\title{
Reconstruction of Nonuniformly Sampled Bandlimited Signals by Means of Time-Varying Discrete-Time FIR Filters
}

\author{
Håkan Johansson and Per Löwenborg \\ Electronics Systems of the Department of Electrical Engineering, Linköping University, 58183 Linköping, Sweden
}

Received 1 September 2004; Revised 20 December 2004; Accepted 6 January 2005

\begin{abstract}
This paper deals with reconstruction of nonuniformly sampled bandlimited continuous-time signals using time-varying discretetime finite-length impulse response (FIR) filters. The main theme of the paper is to show how a slight oversampling should be utilized for designing the reconstruction filters in a proper manner. Based on a time-frequency function, it is shown that the reconstruction problem can be posed as one that resembles an ordinary filter design problem, both for deterministic signals and random processes. From this fact, an analytic least-square design technique is then derived. Furthermore, for an important special case, corresponding to periodic nonuniform sampling, it is shown that the reconstruction problem alternatively can be posed as a filter bank design problem, thus with requirements on a distortion transfer function and a number of aliasing transfer functions. This eases the design and offers alternative practical design methods as discussed in the paper. Several design examples are included that illustrate the benefits of the proposed design techniques over previously existing techniques.
\end{abstract}

Copyright @ 2006 Hindawi Publishing Corporation. All rights reserved.

\section{INTRODUCTION}

Nonuniform sampling occurs in many practical applications either intentionally or unintentionally [1]. An example of intentional nonuniform sampling is found in analog-to-digital conversion where certain time slots are used for calibration and so forth. This can be viewed as if some samples from a uniform grid are discarded which results in a nonuniform grid. Unintentional uniform sampling occurs in, for example, high-speed time-interleaved analog-to-digital converters (ADCs), where static time-skew errors between the different subconverters give rise to a class of periodic nonuniform sampling [2] as exemplified in Figure 1(c).

Regardless whether the continuous-time (CT) signal, say $x_{a}(t)$, has been sampled uniformly (Figure $1(\mathrm{a})$ ), producing the sequence $x(n)=x_{a}(n T)$ or nonuniformly (Figure 1(b)), producing the sequence $x_{1}(n)=x_{a}\left(t_{n}\right)$, it is often desired to reconstruct $x_{a}(t)$ from the generated sequence of numbers. Thus, in the nonuniform-sampling case, it is desired to recover $x_{a}(t)$ from the sequence $x_{1}(n)$. This can, in principle, be done in two different ways. The first way is to reconstruct $x_{a}(t)$ directly from $x_{1}(n)$ through CT reconstruction functions. Although it is known how to do this in principle (see, e.g., $[1,3-7])$, problems arise when it comes to practical implementations. In particular, it is very difficult to practically implement CT functions with high precision. It is therefore desired to use the second way which is to perform the reconstruction in the digital domain, that is, to first recover $x(n)$. One then needs only one conventional digital-toanalog converter (DAC) and a CT filter to obtain $x_{a}(t)$, which are much easier to implement than general complicated CT functions. Recovery of $x(n)$ is also of interest even if $x_{a}(t)$ is not to be reconstructed. For example, in receivers in digital communication systems, $x(n)$ is the final goal.

Recovering $x(n)$ from $x_{1}(n)$ in the digital domain can, in principle, be done by utilizing discrete-time (DT) reconstruction functions obtained through sampling of a corresponding ideal CT reconstruction function. However, these CT reconstruction functions are generally noncausal (twosided) functions, which therefore must be truncated in order to make the corresponding digital reconstruction system practically implementable. This truncation causes reconstruction errors that are not easily controlled. In particular, problems arise when one attempts to approximate perfect reconstruction over the whole frequency range $|\omega T| \leq \pi, \omega T$ being the "DT frequency" variable.

To get around the above mentioned problems, one should (1) assume a slight oversampling and (2) use design techniques that are not based on truncation of infiniteduration functions. In this way one can (1) for a given filter order obtain the best approximation with respect to the frequency region of interest $|\omega T| \leq \omega_{0} T<\pi$ (i.e., for the slightly oversampled case) and (2) for a given allowed approximation error obtain the minimum-order filters meeting 


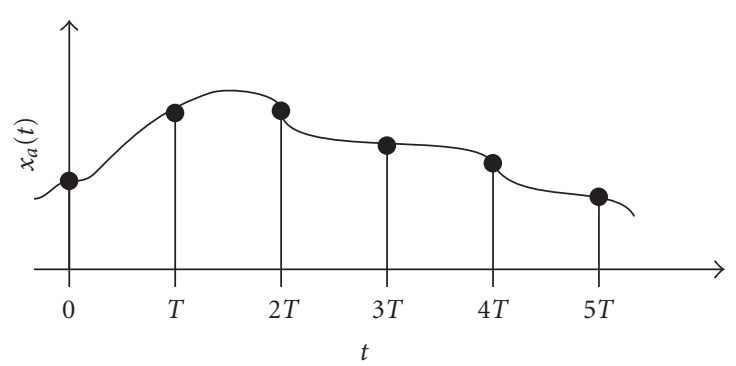

(a)

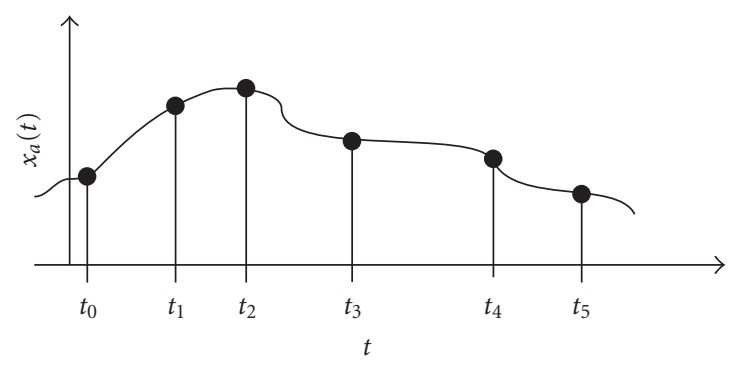

(b)

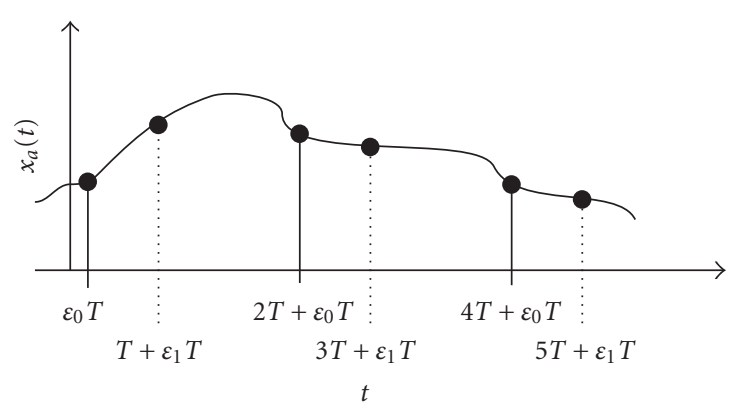

(c)

FIgURE 1: (a) Uniform sampling, (b) nonuniform sampling, and (c) periodic nonuniform sampling.

this criterion. Whereas this approach is known to be beneficial when designing ordinary frequency selective filters [8], it has hitherto not been fully explored as to reconstruction systems. For example, the method introduced in [9] employs causal interpolation functions, and it was observed experimentally that the reconstruction deteriorates when the bandwidth approaches $\pi$, but a theoretical study was not provided. A main theme of this paper is therefore to investigate these issues in detail as outlined in Section 1.1 below. Before embarking on that discussion, it is stressed that, even though oversampling itself is undesired since it generates more samples than necessary for reconstruction according to the Nyquist sampling theorem, it is recognized that a slight oversampling is required in most signal processing systems in order to make them practically implementable. Hence, the assumption that the signals be slightly oversampled is not a disadvantage from the practical point of view (albeit it may be so from a strict theoretical point of view), but instead an advantage or even a necessity in order to obtain a practically implementable high-performance reconstruction system.

\subsection{Purpose of the paper}

The purpose of this paper is three-fold as discussed in the following. The first purpose of the paper is to provide further insights into the problem of reconstructing nonuniformly sampled bandlimited signals using time-varying discretetime FIR filters. To this end, the starting point is a bandlimited CT signal that is nonuniformly sampled and slightly oversampled as to the average sampling frequency, the reason for the latter assumption being as outlined above. It is further assumed that the sampling instances are known. Under these assumptions, a representation of the reconstructed sequence is derived that utilizes a time-frequency function. This representation enables a proper utilization of the oversampling and reduces the reconstruction problem to a design problem that resembles an ordinary filter design problem. Both deterministic and random signals are considered in the paper.

The second purpose of the paper is to introduce design techniques that properly make use of the oversampling that a priori has been assumed. These techniques rely on the timefrequency function discussed above. Both least-square and minimax design techniques will be discussed.

The third purpose of the paper is to show that, for an important special case, corresponding to a certain type of periodic nonuniform sampling, the design problem can be posed as a filter bank (FB) design problem, thus with requirements on a distortion transfer function (that should approximate one) and a number of aliasing transfer functions (that should approximate zero). This formulation offers alternative design methods as will be discussed as well. A part here is also to discuss the relation between the FB formulation derived in this paper and an alternative FB formulation derived in [7].

\subsection{Relation to previous work}

The idea of using time-varying filters for reconstructing nonuniformly sampled signals is not new. As mentioned earlier, this solution is automatically obtained through sampling and truncation of a corresponding ideal CT reconstruction function. Although such a solution may be the best approximation to the ideal solution, it will only be so with respect to the whole frequency range $|\omega T| \leq \pi$. It will not be the best approximation with respect to the frequency region $|\omega T| \leq \omega_{0} T<\pi$, that is, for the slightly oversampled case. The reason is that truncation of infinite-length functions does not handle don't-care bands. A consequence of using designs based on truncation is therefore that the order required to obtain an acceptable reconstruction error in the region $|\omega T| \leq \omega_{0} T<\pi$ may become much higher than actually needed. Using instead the time-frequency function employed in this paper, and the proposed design techniques based on that function, one can utilize the oversampling and obtain minimum-order filters. This will be demonstrated through design examples (Example 4), where the proposed approach is compared to truncated CT Lagrange-based filters $[1,10]$.

As to the special case of periodic nonuniform sampling, it is noted that an FB formulation of this problem has been 
derived earlier in [7], but there is a difference between the formulation in that paper and the one to be derived in this paper. The advantage of using the FB formulation in this paper is that it enables one to directly make use of the design procedure derived for the general case where the sampling is not periodically nonuniform. In this way, the filter orders can be reduced compared to those of the practical filters suggested up to now $[11,12]$ for approximating the ideal ones in [7] (as will be demonstrated in Examples 5 and 8). Finally, it is also pointed out that a special class of fractional-delay reconstructing filters was proposed in [13] for solving the same problem. The advantage of that approach is that the filters need not be redesigned in case the sampling pattern is changed, which is an advantage in real-time implementations. The drawback of the approach in [13] is that a certain amount of additional oversampling must be used. The approach in this paper (as well as that in [12]) overcomes this drawback, but it should be noted that the time-varying filters must be redesigned if the periodic nonuniform sampling pattern is changed during operation for whatever reason.

Finally, it is noted that parts of the work in this paper have been presented at a conference [14] and a workshop [15].

\subsection{Paper outline}

Following this introduction, Sections 2 and 3 consider nonuniform sampling and reconstruction using time-varying discrete-time FIR filters for deterministic signals and random processes, respectively. Based on the results in Section 3, Section 4 introduces a least-square design technique. Section 5 studies the special case of periodic nonuniform sampling. Section 6 discusses the extension from lowpass to bandpass sampling and reconstruction. Finally, Section 7 concludes the paper.

\section{NONUNIFORM SAMPLING AND RECONSTRUCTION OF DETERMINISTIC SIGNALS}

Throughout this paper, it is assumed that the nonuniform sampling of the CT signal $x_{a}(t)$ is done in such a way that the so obtained sequence, say, $x_{1}(n)$, is given by

$$
x_{1}(n)=x_{a}\left(t_{n}\right)
$$

where

$$
t_{n}=n T+\varepsilon_{n} T
$$

with $\varepsilon_{n} T$ representing the distance between the "nonuniform sampling instance" $t_{n}$ and the "uniform sampling instance" $n T$. The average sampling frequency is thus still $1 / T$. It is also assumed that the sampling instances are known and distinct, that is, $t_{n} \neq t_{m}, n \neq m$, and that $t_{n}<t_{m}, n<m$. Furthermore, it is assumed that $x_{a}(t)$ is bandlimited according to

$$
X_{a}(j \omega)=0, \quad 0<\omega_{0}<|\omega|, \omega_{0}<\pi / T
$$

(see also Figure 2(a)). That is, the Nyquist criterion for sampling with a sampling frequency of $1 / T$ without aliasing is fulfilled. In addition, it is for practical reasons assumed that $x_{a}(t)$ is slightly oversampled which means that there is a certain don't-care region between $\omega_{0}$ and $\pi / T$, where the signal contains no frequency components. The above assumption regarding the frequency content of $x_{a}(t)$ is valid for the lowpass (baseband) case which mainly is considered in this paper. However, in Section 6, it is shown that bandpass signals can be handled as well after some minor modifications.

\subsection{Reconstruction}

Given the nonuniform-sampling sequence $x_{1}(n)$, it is here desired to recover the uniform-sampling sequence $x(n)$, that is, to obtain

$$
x(n)=x_{a}(n T), \quad n=\ldots,-2,-1,0,1,2, \ldots
$$

When analyzing and designing a reconstruction system, it is convenient to make use of Poisson's summation formula which relates the Fourier transforms of $x(n)$ and $x_{a}(t) .{ }^{1}$ This formula reads

$$
X\left(e^{j \omega T}\right)=\frac{1}{T} \sum_{r=-\infty}^{\infty} X_{a}\left(j \omega-j \frac{2 \pi r}{T}\right),
$$

where $X\left(e^{j \omega T}\right)$ and $X_{a}(j \omega)$ denote the Fourier transforms of $x(n)$ and $x_{a}(t)$, respectively. Since the spectrum of $x(n)$ is periodic with period $2 \pi$ ( $2 \pi$-periodic) with respect to $\omega T$, it suffices to consider $X\left(e^{j \omega T}\right)$ in the interval $-\pi \leq \omega T \leq \pi$. When (3) holds, it follows from (5) that

$$
X\left(e^{j \omega T}\right)=\frac{1}{T} X_{a}(j \omega), \quad-\pi \leq \omega T \leq \pi
$$

(see also Figure 2(b)). Equation (6) implies that $x_{a}(t)$ can be recovered from $x(n)$. In practice, this is done using a DAC followed by a CT reconstruction filter. It is also noted that $x_{a}(t)$ is oversampled unless $\omega_{0}=\pi / T$.

With $x_{1}(n)$ as input, the reconstruction system generates a new sequence, say $y(n)$. It is desired to achieve $y(n)=$ $x(n)$ because then $x_{a}(t)$ can, due to (6), be recovered using conventional reconstruction methods for uniformly sampled signals. The equality $y(n)=x(n)$ corresponds in the frequency domain to $Y\left(e^{j \omega T}\right)=X\left(e^{j \omega T}\right)$. If these equations hold, the reconstruction system is said to be a perfect reconstruction $(P R)$ system. In practice, PR can generally only be approximated, which implies that the role of any practical reconstruction system is to make $y(n)$ approximate $x(n)$ as well as possible in some sense. In this paper, time-varying discrete-time FIR filters are used for the reconstruction.

\footnotetext{
1 The results derived in this paper hold for bandlimited signals that can be represented with the aid of Fourier transforms. The results hold for real as well as complex signals, but the paper focuses on real signals which explains why the frequency band stretches from $-\omega_{0}$ to $\omega_{0}$ (in the lowpass case) and real reconstruction filters are employed. These filters may be used also for complex signals whose frequency band stretches from, say $\omega_{1}$ to $\omega_{2}, \omega_{1}<\omega_{2}$, by choosing $\omega_{0}=\max \left(\left|\omega_{1}\right|,\left|\omega_{2}\right|\right)$, but they will be overdesigned as they cover a wider band than necessary. In such cases, it may therefore be beneficial to use instead complex reconstruction filters, but this is not considered in this paper.
} 


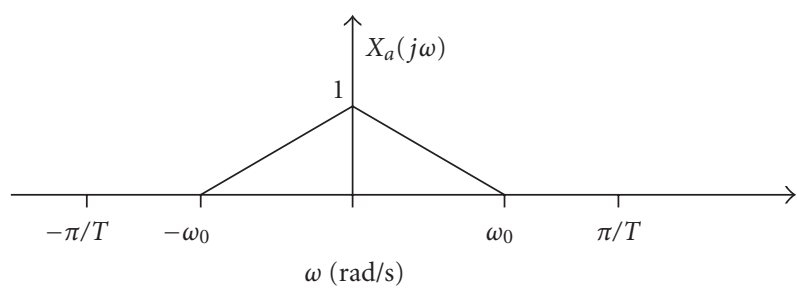

(a)

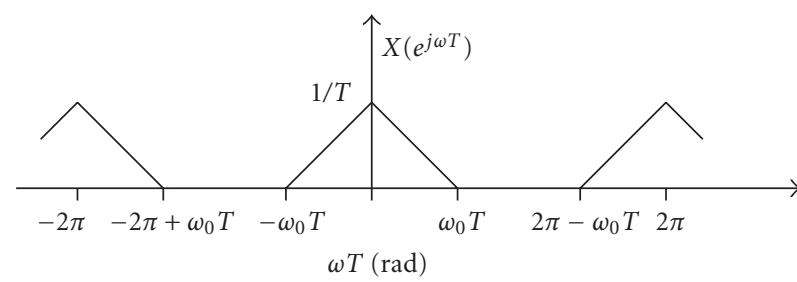

(b)

FIgURE 2: Spectra of a bandlimited signal $x_{a}(t)$ and the sequence $x(n)=x_{a}(n T)$ (uniform sampling).

\subsection{Reconstruction using time-varying discrete-time FIR filters}

In this paper, the reconstruction is performed using a timevarying discrete-time FIR filter characterized by the real impulse responses $h_{n}(k)$ (see Footnote 1). It is assumed here that the order of the FIR filter is $2 \mathrm{~N}$ and thus even. For the analysis and design, it is convenient to let the FIR filter be noncausal and its impulse response is therefore selected to be nonzero for $k=-N,-N+1, \ldots, N$, which means that each $h_{n}(k)$ is centred around the sample that is to be recovered. In a practical implementation, the corresponding causal filter is obtained by simply introducing a delay of $N$ samples. In the odd-order case, one has instead $k=-N,-N+1, \ldots, N-1$, or $k=-N+1,-N+2, \ldots, N$, but that will not change the principles dealt with in this paper. Henceforth, only the evenorder case is therefore considered for the sake of simplicity.

Under the above assumptions, $y(n)$ is now formed according to

$$
y(n)=\sum_{k=-N}^{N} x_{1}(n-k) h_{n}(k)
$$

It is desired to select $h_{n}(k)$ so that $y(n)$ approximates $x(n)$ as close as possible in some sense. To see how to choose $h_{n}(k)$, $x_{1}(n)$ is first written in terms of the inverse Fourier transform of $x_{a}(t)$ by which one obtains, due to (1)-(3),

$$
y(n)=\frac{1}{2 \pi} \int_{-\omega_{0}}^{\omega_{0}} e^{j \omega T \varepsilon_{n}} X_{a}(j \omega) e^{j \omega T n} d \omega .
$$

Inserting (8) into (7), and interchanging the summation and integration, one obtains

$$
y(n)=\frac{1}{2 \pi} \int_{-\omega_{0}}^{\omega_{0}} A_{n}(j \omega T) X_{a}(j \omega) e^{j \omega T n} d \omega,
$$

where

$$
A_{n}(j \omega T)=\sum_{k=-N}^{N} h_{n}(k) e^{-j \omega T\left(k-\varepsilon_{n-k}\right)} .
$$

When (6) holds, (9) can equivalently be written as

$$
y(n)=\frac{1}{2 \pi} \int_{-\omega_{0} T}^{\omega_{0} T} A_{n}(j \omega T) X\left(e^{j \omega T}\right) e^{j \omega T n} d(\omega T) .
$$

Equation (11) represents $y(n)$ with the aid of the functions $A_{n}(j \omega T)$, which can be viewed either as a time-frequency function or an infinite set of frequency functions. Further, $x(n)$ can be expressed in terms of its inverse Fourier transform according to

$$
x(n)=\frac{1}{2 \pi} \int_{-\omega_{0} T}^{\omega_{0} T} X\left(e^{j \omega T}\right) e^{j \omega T n} d(\omega T) .
$$

Comparing (11) with (12), it is seen that perfect reconstruction is obtained if

$$
A_{n}(j \omega T)=1, \quad \omega T \in\left[-\omega_{0} T, \omega_{0} T\right],
$$

for all $n$.

\subsection{Error bound}

Defining the error $e(n)$ as $e(n)=y(n)-x(n)$, one obtains from (11) and (12) that

$$
e(n)=\frac{1}{2 \pi} \int_{-\omega_{0} T}^{\omega_{0} T}\left(A_{n}(j \omega T)-1\right) X\left(e^{j \omega T}\right) e^{j \omega T n} d(\omega T) .
$$

Apparently, $e(n)=0$ in the PR case since, then, $A_{n}(j \omega T)=1$. In practice, $A_{n}(j \omega T)$ can generally only approximate one in the frequency range of interest. The goal is then to determine the coefficients $h_{n}(k)$ so that the error $e(n)$ is minimized according to some criterion. A problem is that $e(n)$ does not only depend on $h_{n}(k)$, but also on $X\left(e^{j \omega T}\right)$ which means that one generally must have knowledge about the input signal spectrum in order to determine $h_{n}(k)$ in the best possible way. If one does not have complete knowledge about $X\left(e^{j \omega T}\right)$, which often is the case in practice, one has to accept a suboptimum solution instead. To this end, it is often convenient to make use of the $L_{p}$-norm which, for $X\left(e^{j \omega T}\right)$, is given by

$$
\left\|X\left(e^{j \omega T}\right)\right\|_{p}=\sqrt[p]{\frac{1}{2 \pi} \int_{-\pi}^{\pi}\left|X\left(e^{j \omega T}\right)\right|^{p} d(\omega T)} .
$$

Applying now the triangle inequality for integrals on (14), one obtains

$$
\begin{aligned}
|e(n)| & \leq \frac{1}{2 \pi} \int_{-\pi}^{\pi}\left|A_{n}(j \omega T)-1\right|\left|X\left(e^{j \omega T}\right)\right| d(\omega T) \\
& \leq\left\|A_{n}(j \omega T)-1\right\|_{\infty}\left\|X\left(e^{j \omega T}\right)\right\|_{1} .
\end{aligned}
$$


Based on (16), one can draw the conclusion that minimizing $\left\|A_{n}(j \omega T)-1\right\|_{\infty}$ is appropriate for narrow band signals. To see this, consider the sinusoidal signal

$$
x(n)=X_{m} \sin (\omega T n+\phi)
$$

For this signal, only the $L_{1}$-norm exists and, from the definition in (15), one gets

$$
\left\|X\left(e^{j \omega T}\right)\right\|_{1}=X_{m}
$$

From (16) and (18), it now follows that

$$
|e(n)| \leq\left\|A_{n}(j \omega T)-1\right\|_{\infty} X_{m}
$$

This means that, for any sinusoidal input, with the angular frequency $\omega \in\left[-\omega_{0}, \omega_{0}\right]$ and amplitude $X_{m}$, the error $e(n)$ is bounded by (19). The next section discusses how to design $A_{n}(j \omega T)$ so as to minimize $\left\|A_{n}(j \omega T)-1\right\|_{\infty}$.

\subsection{Filter design}

By utilizing the representation of $y(n)$ in (11), the design of the reconstructing system reduces to the problem of minimizing the "size" of $A_{n}(j \omega T)-1$, according to some criterion. This corresponds to designing an FIR filter with noninteger delays. ${ }^{2}$ This issue has been considered earlier in the literature [16-18], but mainly for frequency-selective filters, not reconstruction of nonuniformly sampled signals. There are mainly two features that differ the signal reconstruction context in this paper from those contexts.

First, there is only one band of interest here, namely, the passband region $\omega T \in\left[-\omega_{0} T, \omega_{0} T\right], \omega_{0} T<\pi$, which means that the problems associated with designing frequency selective filters with noninteger delays [16-18] are circumvented. In particular, it is difficult to obtain such frequency selective filters with arbitrary passband and stopband regions throughout the whole region $\omega T \in[-\pi, \pi]$. This difficulty does not appear here, at least not for reasonable values of $\varepsilon_{n} T$, as $A_{n}(j \omega T)$ are to be allpass (up to $\omega_{0} T<\pi$ ), not frequency selective.

Second, a new $A_{n}(j \omega T)$ has to be designed here for each value of $n .^{3}$ This means that one should employ design techniques that are easily implemented. This is especially the case for real-time applications that cannot house costly and time-consuming online filter design. This is a problem when designing $A_{n}(j \omega T)$ for narrow-band signals. As discussed earlier, it is in this case appropriate to determine each $h_{n}(k)$ so that each $\left\|A_{n}(j \omega T)-1\right\|_{\infty}$ is minimized in the frequency region $\omega T \in\left[-\omega_{0} T, \omega_{0} T\right], \omega_{0} T<\pi$. This is a convex optimization problem [19], and $h_{n}(k)$ can be found by using a standard minimax design technique to this

\footnotetext{
2 The noninteger delays are merely a consequence of the nonuniform sampling and the problem formulation. Thus, they are not actually implemented which naturally would cause problems.

${ }^{3}$ In the case of periodic nonuniform sampling, it suffices to design only a few functions, see Section 5.
}

end. However, minimax techniques are generally costly and time-consuming to implement and not suitable for online design. To achieve feasible online design, one may adopt a least-square approach where $h_{n}(k)$ can be determined analytically through matrix inversion. Although a least-square solution is not the optimum solution to the problem of minimizing $\left\|A_{n}(j \omega T)-1\right\|_{\infty}$, it is indeed a good approximation. One can therefore satisfy requirements on $\left\|A_{n}(j \omega T)-1\right\|_{\infty}$, even when least-square design methods are adopted instead of minimax design techniques. The price to pay is a slightly increased filter order, but it may be necessary to accept this in order to avoid complicated online design. Section 4 introduces a least-square approach that can be used for this purpose.

\subsection{Special case of multi-sine input signals}

When the input signal $x_{a}(t)$ has a discrete spectrum containing $2 N+1$ complex sinusoidals with angular frequencies $\omega_{p}, p=-N,-N+1, \ldots, N$, the "uniform samples" $x_{a}(n T)$ can be computed exactly from the "nonuniform samples" $x_{a}\left(n T+\varepsilon_{n} T\right)$. Making use of the representation of $y(n)$ in (11), it is obvious that this is achieved by satisfying $A_{n}(j \omega T)=1$ for $\omega=\omega_{p}$. This results in a system of $2 N+1$ linear equations which can be solved uniquely provided that the corresponding matrix is nonsingular. To be precise, $h_{n}(k)$ are obtained from

$$
\mathbf{h}_{n}=\mathbf{D}_{n}^{-1} \mathbf{c}
$$

where

$$
\begin{gathered}
\mathbf{h}_{n}=\left[\begin{array}{cccc}
h_{n}(-N) & h_{n}(-N+1) & \cdots & h_{n}(N)
\end{array}\right]^{T}, \\
\mathbf{c}=\left[\begin{array}{llll}
1 & 1 & \cdots & 1
\end{array}\right]^{T},
\end{gathered}
$$

and each $\mathbf{D}_{n}$ is a $2 N+1 \times 2 N+1$ matrix with the entries $d_{n, p k}$, $p, k=-N,-N+1, \ldots, N$, given by

$$
d_{n, p k}=e^{-j \omega_{p} T\left(k-\varepsilon_{n-k}\right)} .
$$

Nonsingularity cannot be guaranteed in general, but indeed for many cases. One important such case is that of uniformly spaced frequencies according to

$$
\omega_{p} T=\frac{2 \pi p}{2 N+1}
$$

which covers real input signals. In this case, $\mathbf{D}_{n}$ can be written as

$$
\mathbf{D}_{n}=\mathbf{D}_{n 1} \mathbf{D}_{n 2}
$$

where each $\mathbf{D}_{n 1}$ is a Vandermonde matrix with entries $d_{n, q k}^{(1)}$, $q=0,1, \ldots, 2 N, k=-N,-N+1, \ldots, N$, and each $\mathbf{D}_{n 2}$ is a diagonal matrix with diagonal elements $d_{n, k k}^{(2)}, k=-N,-N+$ $1, \ldots, N$, given by

$$
\begin{aligned}
& d_{n, q k}^{(1)}=e^{-j(2 \pi q /(2 N+1))\left(k-\varepsilon_{n-k}\right)}, \\
& d_{n, k k}^{(2)}=e^{-j(2 \pi N /(2 N+1))\left(k-\varepsilon_{n-k}\right)} .
\end{aligned}
$$


As $\mathbf{D}_{n 1}$ is Vandermonde, and $\left|\operatorname{det} \mathbf{D}_{n 2}\right|=1$, nonsingularity of $\mathbf{D}_{n}$ is in this case guaranteed if $e^{-j 2 \pi\left(k-\varepsilon_{n-k}\right) / 2 N+1}$ are distinct. This is, for example, trivially satisfied when $\left|\varepsilon_{n-k}\right|<$ 0.5 .

It is stressed that, when the frequencies are equally spaced according to (23), and more generally when they satisfy $\omega_{p}=$ $-\omega_{-p}$, the impulse responses $h_{n}(k)$ become real-valued, regardless whether the input signal is real (in which case the sum of each pair of $B_{p} \exp \left(j \omega_{p} t\right)$ and $B_{-p} \exp \left(j \omega_{-p} t\right)$ is a real-valued sinusoidal) or complex. This is because (10) in this case equals one for $\omega=\omega_{p}$ as well as $\omega=-\omega_{p}$, which shows that the conjugate of $h_{n}(k)$ is a solution if $h_{n}(k)$ so is. But since the solution is unique (provided $\mathbf{D}_{n}$ are nonsingular), it follows that $h_{n}(k)$ are real-valued. When the input signal $x_{a}(t)$ has a discrete spectrum containing $2 N+1$ frequency components, perfect reconstruction is thus possible when using real $2 \mathrm{~N}$ th-order time-varying FIR filters. For more general bandlimited signals, only approximately perfect reconstruction is feasible, as discussed earlier in this section.

\section{NONUNIFORM SAMPLING AND RECONSTRUCTION OF RANDOM PROCESSES}

Consider now the case where $x_{a}(t)$ is a random wide-sense stationary (WSS) process. ${ }^{4}$ It is assumed that $x_{a}(t)$ is bandlimited according to

$$
R_{X_{a} X_{a}}(j \omega)=0, \quad 0<\omega_{0}<|\omega|, \omega_{0}<\pi / T,
$$

where $R_{X_{a} X_{a}}(j \omega)$ denotes the power spectral density function of $x_{a}(t)$ which is the Fourier transform of the autocorrelation function $r_{X_{a} X_{a}}(\tau)=E\left\{x_{a}(t) x_{a}^{*}(t-\tau)\right\}$, where $E\{x\}$ denotes the expected value of $x$ and $*$ denotes conjugate. With $x(n)=$ $x_{a}(n T)$, one has (compare with (5))

$$
R_{X X}\left(e^{j \omega T}\right)=\frac{1}{T} \sum_{r=-\infty}^{\infty} R_{X_{a} X_{a}}\left(j \omega-j \frac{2 \pi r}{T}\right)
$$

where $R_{X X}\left(e^{j \omega T}\right)$ denotes the power spectral density function of $x(n)$ which is the Fourier transform of the autocorrelation sequence $r_{X X}(k)=E\left\{x(n) x^{*}(n-k)\right\}=r_{X_{a} X_{a}}(k T)$. In particular, for the bandlimited case in (26), (27) implies

$$
R_{X X}\left(e^{j \omega T}\right)=\frac{1}{T} R_{X_{a} X_{a}}(j \omega), \quad-\pi \leq \omega T \leq \pi .
$$

For random processes, it is common to use the expected quadratic error $E\left\{|e(n)|^{2}\right\}$, where $e(n)=y(n)-x(n)$, as a measure of the reconstruction error. To derive a useful expression of this error, it is first noted that $E\left\{|e(n)|^{2}\right\}$ in

\footnotetext{
${ }^{4}$ In the literature, it is common to use capital letters to denote random processes, like $X_{a}(t)$ and so forth. However, in order to be able to directly make use of the expressions derived for the deterministic signals, small letters are used in this paper, that is, $x_{a}(t)$ and so forth.
}

expanded form is

$$
\begin{aligned}
E\left\{|e(n)|^{2}\right\}= & E\left\{|y(n)|^{2}\right\}-E\left\{y(n) x^{*}(n)\right\} \\
& -E\left\{y^{*}(n) x(n)\right\}+E\left\{|x(n)|^{2}\right\} .
\end{aligned}
$$

From (1) and (7), and the fact that the operator $E$ and summation can be interchanged, it follows that the first term in (29) becomes

$$
\begin{aligned}
& E\left\{|y(n)|^{2}\right\} \\
&=\sum_{k=-N}^{N} \sum_{p=-N}^{N} h_{n}(k) h_{n}(p) \\
& \quad \times r_{X_{a} X_{a}}\left(p T-k T+\varepsilon_{n-k} T-\varepsilon_{n-p} T\right) .
\end{aligned}
$$

Since $r_{X_{a} X_{a}}(\tau)$ and $R_{X_{a} X_{a}}(j \omega)$ form a Fourier transform pair, and $R_{X_{a} X_{a}}(j \omega)$ is bandlimited, (30) can alternatively be expressed as

$$
\begin{aligned}
& E\left\{|y(n)|^{2}\right\} \\
& =\sum_{k=-N}^{N} \sum_{p=-N}^{N} h_{n}(k) h_{n}(p) \\
& \quad \times \frac{1}{2 \pi} \int_{-\omega_{0}}^{\omega_{0}} R_{X_{a} X_{a}}(j \omega) e^{-j \omega T\left(p-k+\varepsilon_{n-k}-\varepsilon_{n-p}\right)} d \omega .
\end{aligned}
$$

By interchanging the integration and summations, and utilizing (10), one finally ends up with

$$
E\left\{|y(n)|^{2}\right\}=\frac{1}{2 \pi} \int_{-\omega_{0}}^{\omega_{0}} R_{X_{a} X_{a}}(j \omega)\left|A_{n}(j \omega T)\right|^{2} d \omega,
$$

where $A_{n}(j \omega T)$ are again given by (10).

As to the second and third terms in (29), one obtains in a similar way

$$
\begin{aligned}
& E\left\{y(n) x^{*}(n)\right\}=\frac{1}{2 \pi} \int_{-\omega_{0}}^{\omega_{0}} R_{X_{a} X_{a}}(j \omega) A_{n}(j \omega T) d \omega, \\
& E\left\{y^{*}(n) x(n)\right\}=\frac{1}{2 \pi} \int_{-\omega_{0}}^{\omega_{0}} R_{X_{a} X_{a}}(j \omega) A_{n}(-j \omega T) d \omega .
\end{aligned}
$$

Due to the fact that $R_{X_{a} X_{a}}(j \omega)$ is real, because $r_{X_{a} X_{a}}(\tau)$ is Hermitian symmetric, that is, $r_{X_{a} X_{a}}(\tau)=r_{X_{a} X_{a}}^{*}(-\tau)$, it follows from (33) that

$$
\begin{aligned}
& E\left\{y(n) x^{*}(n)\right\}+E\left\{y^{*}(n) x(n)\right\} \\
& =2 \operatorname{Re}\left\{E\left\{y(n) x^{*}(n)\right\}\right\} \\
& =\frac{1}{\pi} \int_{-\omega_{0}}^{\omega_{0}} R_{X_{a} X_{a}}(j \omega) \operatorname{Re}\left\{A_{n}(j \omega T)\right\} d \omega
\end{aligned}
$$


provided that $A_{n}(j \omega T)$ are Hermitian symmetric, that is, $A_{n}(j \omega T)=A_{n}^{*}(-j \omega T)$. It is seen in (10) that this is always the case when $h_{n}(k)$ are real.

Finally, for the fourth term in (29), one has $E\left\{|x(n)|^{2}\right\}=$ $E\left\{\left|x_{a}(n T)\right|^{2}\right\}=r_{X_{a} X_{a}}(0)$, which means that one can write

$$
E\left\{|x(n)|^{2}\right\}=\frac{1}{2 \pi} \int_{-\omega_{0}}^{\omega_{0}} R_{X_{a} X_{a}}(j \omega) d \omega
$$

Combining the above results, and utilizing (28) together with the fact that $1+|x|^{2}-2 \operatorname{Re}\{x\}=|x-1|^{2}$, one ends up with

$$
E\left\{|e(n)|^{2}\right\}=\int_{-\omega_{0} T}^{\omega_{0} T} R_{X X}\left(e^{j \omega T}\right)\left|A_{n}(j \omega T)-1\right|^{2} d(\omega T) .
$$

Equation (36) indicates that the least-square design technique that will be introduced in the subsequent section is appropriate when it is desired to minimize the error power.

\section{LEAST-SQUARE DESIGN}

This section introduces a least-square design technique for $A_{n}(j \omega T)$ that is attractive because $h_{n}(k)$ can then be determined analytically through matrix inversion. It follows from (36) that it is appropriate to use the following error power functions $P_{n}$ for this purpose:

$$
P_{n}=\frac{1}{2 \pi} \int_{-\omega_{0} T}^{\omega_{0} T} W^{2}(\omega T)\left|A_{n}(j \omega T)-1\right|^{2} d(\omega T) .
$$

The function $W^{2}(\omega T)$ is a positive weighting function that may or may not include the input signal spectrum (see (36)). Inserting (10) into (37), it follows, after some algebraic manipulations, that $P_{n}$ can be written in matrix form

$$
P_{n}=\mathbf{h}_{n}^{T} \mathbf{S}_{n} \mathbf{h}_{n}+\mathbf{c}_{n}^{T} \mathbf{h}_{n}+C
$$

where

$$
\begin{gathered}
\mathbf{h}_{n}=\left[\begin{array}{llll}
h_{n}(-N) & h_{n}(-N+1) & \cdots & h_{n}(N)
\end{array}\right]^{T}, \\
\mathbf{c}_{n}=\left[\begin{array}{llll}
c_{n,-N} & c_{n,-N+1} & \cdots & c_{n, N}
\end{array}\right]^{T},
\end{gathered}
$$

with $c_{n, k}, k=-N,-N+1, \ldots, N$, being

$$
c_{n, k}=-\frac{1}{\pi} \int_{-\omega_{0} T}^{\omega_{0} T} W^{2}(\omega T) \cos \left(\omega T\left(k-\varepsilon_{n-k}\right)\right) d(\omega T) .
$$

Further, $\mathbf{S}_{n}$ are $2 N+1 \times 2 N+1$ symmetric (provided $W^{2}(\omega T)$ is symmetric around $\omega T=0)$ and positive definite matrices with entries $s_{n, k p}, k, p=-N,-N+1, \ldots, N$, given by

$$
s_{n, k p}=\frac{1}{2 \pi} \int_{-\omega_{0} T}^{\omega_{0} T} W^{2}(\omega T) e^{j \omega T\left(k-p-\varepsilon_{n-k}+\varepsilon_{n-p}\right)} d(\omega T) .
$$

Finally, the constant $C$ is given by

$$
C=\frac{1}{2 \pi} \int_{-\omega_{0} T}^{\omega_{0} T} W^{2}(\omega T) d(\omega T)
$$

For each $n$, the values of $h_{n}(k)$ that minimize the function $P_{n}$ are obtained by setting the partial derivatives of $P_{n}$ with respect to $h_{n}(k)$ to zero and solving for $h_{n}(k)$. With $P_{n}$ in the form of (38), the solution to this problem is immediately obtained as

$$
\mathbf{h}_{n}=-0.5 \mathbf{S}_{n}^{-1} \mathbf{c}_{n}
$$

In order to compute $\mathbf{h}_{n}$ in (43), it is necessary to compute the integrals in (40) and (41). Depending on the weighting function $W(\omega T)$, the integrals may have to be computed numerically. When $W(\omega T)$ is a simple function, they may however be computed analytically. For example, in the simplest case where $W(\omega T)=1, c_{n, k}$ and $s_{n, k p}$ become

$$
\begin{aligned}
& c_{n, k}=\left\{\begin{array}{l}
-\frac{2 \omega_{0} T}{\pi}, \quad k-\varepsilon_{n-k}=0, \\
-\frac{2 \sin \left[\omega_{0} T\left(k-\varepsilon_{n-k}\right)\right]}{\pi\left(k-\varepsilon_{n-k}\right)}, \quad k-\varepsilon_{n-k} \neq 0,
\end{array}\right. \\
& s_{n, k p}= \begin{cases}\frac{\omega_{0} T}{\pi}, \quad k=p, \\
\frac{\sin \left[\omega_{0} T\left(k+\varepsilon_{n-p}-p-\varepsilon_{n-k}\right)\right]}{\pi\left(k+\varepsilon_{n-p}-p-\varepsilon_{n-k}\right)}, \quad k \neq p .\end{cases}
\end{aligned}
$$

\subsection{Design examples}

This subsection illustrates through design examples some properties of the proposed least-square design technique and, at the same time, some properties of nonuniform sampling and reconstruction.

Example 1. Figure 3 illustrates how $A_{n}(j \omega T)-1$ reduces as the filter order increases using the least-square design technique introduced above. The sampling instances are here as in (2), with $\varepsilon_{n} T=0.2 \times(-1)^{n}$, whereas $\omega_{0} T=0.9 \pi$.

Example 2. To further illustrate that the reconstruction improves as the filter order increases, Figure 4 plots the reconstruction error versus filter order for $\omega_{0} T=0.9 \pi$, and 


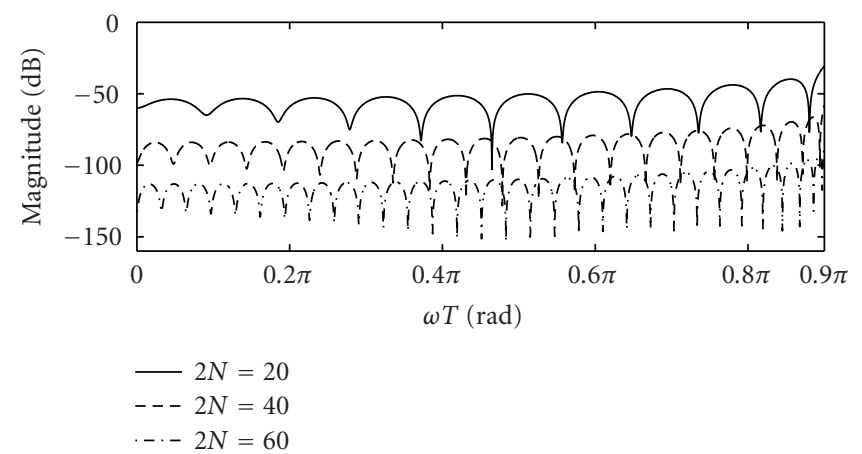

Figure 3: Magnitude $\left(20 \log _{10}\left|A_{n}-1\right|\right.$ for even $\left.n\right)$ for the proposed least-square filters of order $2 N$ (Example 1).

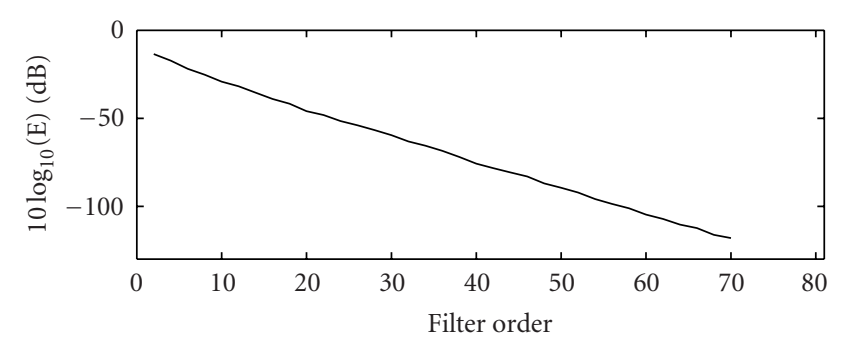

Figure 4: Reconstruction error versus filter order (Example 2).

with $\varepsilon_{n} T$ being randomly chosen numbers in the interval $(-0.45 T, 0.45 T) .^{5}$ For each filter order, the reconstruction error has been computed by taking the average over a large number of designs, each one corresponding to a randomly chosen set of $\left|\varepsilon_{n} T\right|{ }^{6}$

Example 3. Figure 5 illustrates (for the same sampling pattern and randomization as that used in Example 2) how the filter order increases with the bandwidth. It is seen that the order increases rapidly as the "DT bandwidth" $\omega_{0} T$ approaches the full bandwidth $\pi$. This shows that it is advisable to use a slight oversampling of, say, 10-20\%. Figure 6 shows how the filter order increases with increasing maximum $\left|\varepsilon_{n} T\right|$. This illustrates that "the more nonuniform the sampling pattern is," the more difficult it is to reconstruct the signals.

\footnotetext{
${ }^{5}$ In the literature, one sometimes finds the assertion that $\left|\varepsilon_{n} T\right|<0.25 T$ must be fulfilled to enable reconstruction but this is generally not required. In particular, when a slight oversampling is adopted, one circumvents many of the problems associated with reconstruction of critically sampled signals. It should be noted, however, that when two adjacent sampling points approach each other (which may occur if $\left|\varepsilon_{n} T\right|$ are too large), the corresponding matrix $\mathbf{S}_{n}$ in (38) becomes close to singular which causes problems as to invertibility. When two sampling points coincide, $\boldsymbol{S}_{n}$ is singular.

${ }^{6}$ The reconstruction error is here computed as mean $E\left\{P_{n}\right\}$ for $W(\omega T)=$ 1. With a constant $R_{X X}\left(e^{j \omega T}\right)$, the signal-to-noise ratio (SNR) is then $\mathrm{SNR}=10 \log _{10}\left(\omega_{0} T / \pi\right)-10 \log _{10}\left(\right.$ mean $\left.E\left\{P_{n}\right\}\right)$.
}

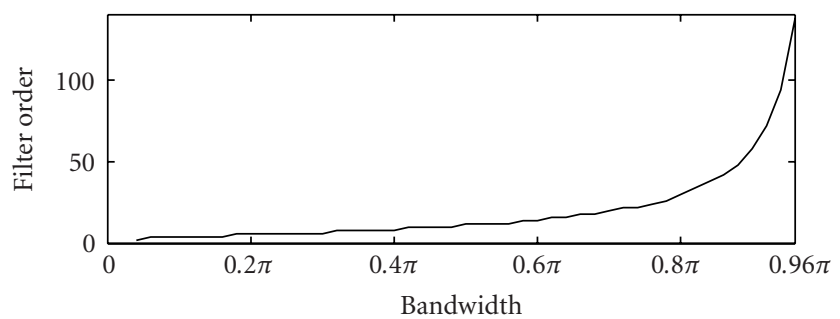

FIGURE 5: Filter order versus bandwidth for a reconstruction error of $-100 \mathrm{~dB}$ (Example 3).

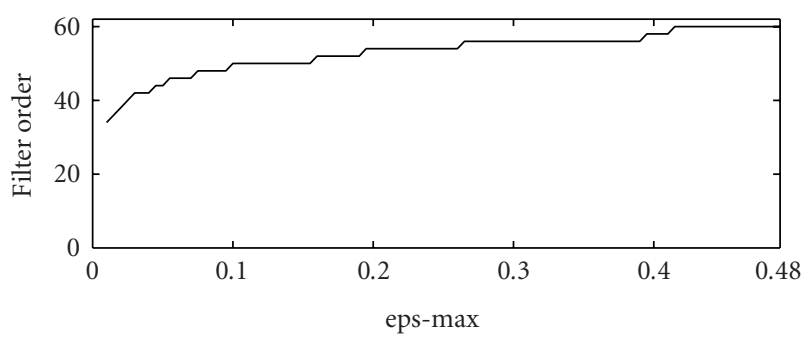

FIGURE 6: Filter order versus maximum sampling instance deviation $\left|\varepsilon_{n} T\right|<$ eps-max for a reconstruction error of $-100 \mathrm{~dB}$ (Example 3 ).

Example 4. Finally, this section compares the proposed leastsquare filters with truncated CT Lagrange filters $[1,10]$. In the special case where $\varepsilon_{n} T$ are constant, such filters are maximally flat fractional-delay time-invariant FIR filters [20]. As such, they are very good at low frequencies, but poor at higher frequencies. The same holds true in the general case where $\varepsilon_{n} T$ are not constant. That is, using Lagrangebased time-varying FIR filters for recovering nonuniformly sampled bandlimited signals, the reconstruction is good at low frequencies, but deteriorates rapidly with increasing frequency. This means that the order will be very high in order to obtain an acceptable error when $\omega_{0} T$ is close to $\pi$. This is illustrated in Figure 7 for the same sampling pattern as in Example 1. It is seen that the reconstruction error is extremely small at lower frequencies, and that it deteriorates rapidly above a certain frequency that depends on the filter order. ${ }^{7}$ It is also seen that the filter order increases rapidly as $\omega_{0} T$ increases. This means that the Lagrange-based filters are not really an option in practice for larger $\omega_{0} T$. Using instead the proposed least-square design technique, the reconstruction error is more evenly distributed over the frequency range $\left[-\omega_{0} T, \omega_{0} T\right]$, which means that much larger $\omega_{0} T$ can be handled without any problems, as illustrated earlier in

\footnotetext{
${ }^{7}$ For lower frequencies, the quantity $20 \log _{10}\left|A_{n}-1\right|$ decays towards minus infinity (in $\mathrm{dB}$ ) when using Lagrange-based reconstruction filters. The bound of $-300 \mathrm{~dB}$, seen in Figure 7, is due to finite-precision effects in Matlab.
} 


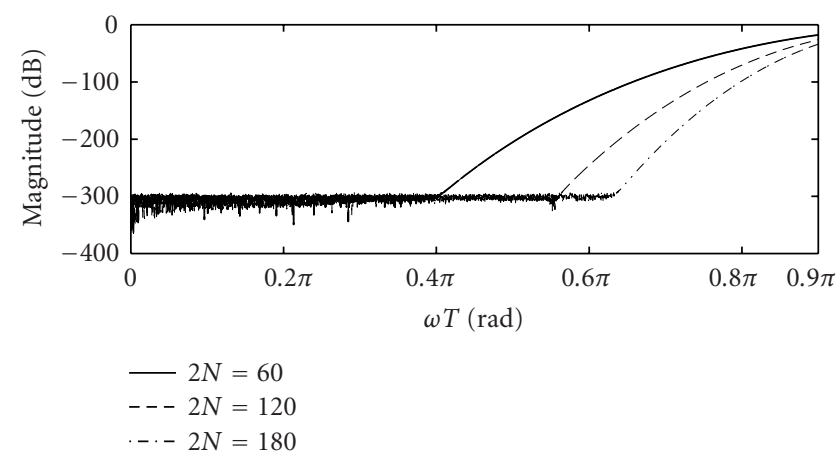

FIgURE 7: Magnitude $\left(20 \log _{10}\left|A_{n}-1\right|\right.$ for even $\left.n\right)$ using Lagrangebased reconstruction filters of order $2 N$ (Example 4).

Figure 3. It also means that, for a given error power, the order of the Lagrange-based filters for larger values of $\omega_{0} T$ become much higher than that of the proposed filters. This is seen in Figure 8, which illustrates how the filter order increases with bandwidth for a reconstruction error of $-60 \mathrm{~dB}$.

\section{PERIODIC NONUNIFORM SAMPLING}

When the "nonuniform sampling deviation sequence" $\varepsilon_{n} T$ exhibits periodicity according to

$$
\varepsilon_{n} T=\varepsilon_{n+M} T
$$

for all $n$, the sampling is said to be periodic nonuniform sampling with period $M$. An example is given in Figure $1(\mathrm{c})$, with period $M=2, \varepsilon_{0} T=\varepsilon_{2 m} T$, and $\varepsilon_{1} T=\varepsilon_{2 m+1} T$, for all integers $m$.

When $\varepsilon_{n} T$ satisfies (46), it is obvious that the timevarying impulse response $h_{n}(k)$ exhibits the same periodicity, that is,

$$
h_{n}(k)=h_{n+M}(k)
$$

for all $n$. In this case, one can easily establish that $y(n)$ in (9), and thus $y(n)$ in Figure $9(\mathrm{a})$, is identical to the output $y(n)$ in the maximally decimated FB shown in Figure 9(b) [21]. That is, the output is obtained as the output of a multirate $\mathrm{FB}$ with the analysis filters $z^{n} H_{n}(z)$, with $H_{n}(z)$ being the transfer function of $h_{n}(k)$, and with the trivial synthesis filters $F_{n}(z)=z^{-n}$. Although this FB structure describes how the reconstruction is actually implemented, it is less suitable for the analysis and design because the input is $x_{1}(n)$, whereas the output $y(n)$ is to approximate $x(n)$ not $x_{1}(n)$, which would be the case in a conventional FB.

However, it is possible to make use of a regular FB structure for easing the analysis and design of the reconstructing system. To see this, it is first observed that the output $y(n)$

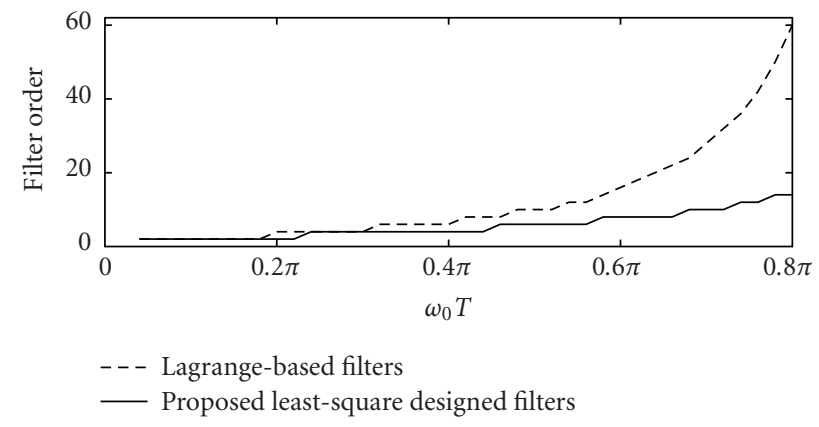

FIGURE 8: Filter order versus bandwidth for a reconstruction error of $-60 \mathrm{~dB}$ (Example 4).

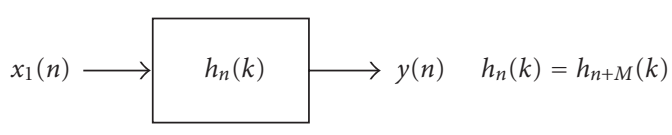

(a)

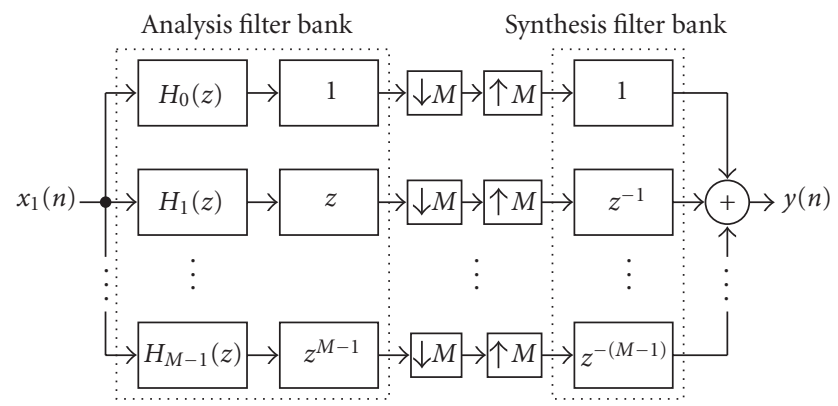

(b)

FIGURE 9: Two equivalent representations of the reconstruction system: (a) time-varying filter and (b) multirate filter bank.

from the time-varying filter $g_{n}(k)=g_{n+M}(k)$, with $x(n)$ as input, is

$$
y(n)=\sum_{k=-N}^{N} x(n-k) g_{n}(k)
$$

In accordance with earlier discussions, $y(n)$ in (48), and thus $y(n)$ in Figure $10(\mathrm{a})$, is identical to the output $y(n)$ in the maximally decimated FB shown in Figure 10(b), with $G_{n}(z)$ being the transfer function of $g_{n}(k)$. Further, utilizing the inverse Fourier transform, it follows that $y(n)$ alternatively be expressed as

$$
y(n)=\int_{-\omega_{0} T}^{\omega_{0} T} G_{n}\left(e^{j \omega T}\right) X\left(e^{j \omega T}\right) e^{j \omega T n} d(\omega T) .
$$




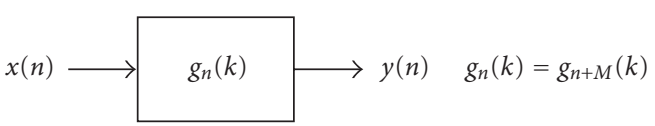

(a)

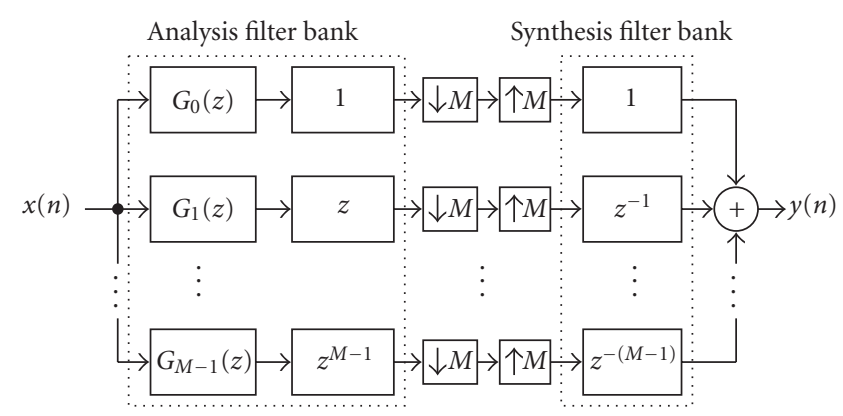

(b)

Figure 10: Two equivalent representations used for analysis and design of the reconstruction system: (a) time-varying filter and (b) multirate filter bank.

Comparing (49) with (11), it is seen that the outputs in the two cases are identical provided that $G_{n}\left(e^{j \omega T}\right)$ satisfy ${ }^{8}$

$$
G_{n}\left(e^{j \omega T}\right)=A_{n}(j \omega T), \quad \omega T \in\left[-\omega_{0} T, \omega_{0} T\right] .
$$

Hence, when the sampling is periodically nonuniform, the reconstruction using time-varying filters can be conveniently represented by the FB in Figure 10(b), with analysis filters as given by (50) and (10). The design problem can in this case be posed as an FB design problem as outlined later in Section 5.3. It is stressed though that the FB in Figure 10(b) is used only with the purpose of easing the analysis and design of the reconstructing system. That is, $y(n)$ is not obtained by implementing this FB which is obvious because that assumes that we already have available the "uniform samples" $x(n)$, which are precisely the samples we want to recover from the "nonuniform samples" $x_{1}(n)$. The output $y(n)$ is naturally still obtained as in (7), and thus as the output in Figure 9.

\subsection{Relation to the FB representation in [7]}

An FB formulation of this problem has been derived earlier in [7], but there are differences between the formulation in that paper and the one in this paper. In [7], it is observed that the nonuniformly sampled signal can be expressed with the aid of a regular decimated analysis filter bank, with analysis filters that are ideal fractional-delay filters determined by the sampling instances and thus fixed, whereas the reconstruction is done via properly designing a

\footnotetext{
${ }^{8}$ It should be noted here that $G_{n}\left(e^{j \omega T}\right)$ are the periodic extensions of $A_{n}(j \omega T)$ since $G_{n}\left(e^{j \omega T}\right)$ are $2 \pi$-periodic (i.e., periodic functions with period $2 \pi$ with respect to $\omega T)$ as they are frequency responses of DT filters whereas $A_{n}(j \omega T)$ are not (see (10)).
}

synthesis filter bank, whose filters $\left(F_{n}(z)\right.$ in Figure 11) ideally are multilevel filters. In this paper, the FB formulation contains instead fixed and trivial synthesis filters (pure delays), whereas the analysis filters are to be designed. Another difference is that the analysis filters are here unconventional in the sense that they make use of noninteger delays (see (10) and Footnote 2). A third difference lies in the implementation. Using time-varying FIR filters, and thus the FB structure in this paper (see Figure 9), each output sample is computed using a segment (of length $2 N+1$ for $2 N$ th-order filters) of consecutive samples in the input sequence $x_{1}(n)$, which are weighted with the impulse response values $h_{n}(k)$. Using instead the FB in [7] (Figure 11), the input sequence is divided into $M$ subsequences according to $x_{1 p}(n)=x_{1}(M n+p)$ for $p=0,1, \ldots, M-1$. These subsequences are then upsampled and fed into the synthesis filters $F_{n}(z)$.

The two different FB representations are, however, closely related and it is easy to start with one of them and derive the other. One can therefore make use of either one when designing and implementing the reconstruction system. The advantage of using the FB formulation in this paper is that the design thereby is simplified. This is done by directly making use of the design procedure derived for the general case, where the sampling is not periodically nonuniform. In the design procedures proposed in $[11,12]$ for the general FB formulation in [7], the ideal and fixed fractional-delay analysis filters are approximated (to enable the design), which is a drawback in the sense that the filter orders of the reconstructing synthesis filters thereby become higher than necessary. Making use of the FB formulation in this paper, together with the proposed design technique, the filter orders can be reduced substantially as will be demonstrated later in Section 5.3.

Given $H_{n}(z)$, the synthesis filters $F_{n}(z)$ are readily obtained by utilizing polyphase decomposition [21] of the filters $z^{n} H_{n}(z)$ by which one can write

$$
\left[\begin{array}{c}
H_{0}(z) \\
z H_{1}(z) \\
\vdots \\
z^{M-1} H_{M-1}(z)
\end{array}\right]=\mathbf{R}\left(z^{M}\right)\left[\begin{array}{c}
1 \\
z \\
\vdots \\
z^{M-1}
\end{array}\right]
$$

where

$$
\mathbf{R}(z)=\left[\begin{array}{cccc}
P_{00}(z) & P_{01}(z) & \cdots & P_{0, M-1}(z) \\
P_{10}(z) & P_{11}(z) & \cdots & P_{1, M-1}(z) \\
\vdots & \vdots & \ddots & \vdots \\
P_{M-1,0}(z) & P_{M-1,1}(z) & \cdots & P_{M-1, M-1}(z)
\end{array}\right]
$$

with $P_{n m}(z)$ denoting the $m$ th polyphase component of $z^{n} H_{n}(z)$. The matrix $\mathbf{R}$ is the polyphase component matrix for the analysis filter bank. From an input-output point of view, this matrix may equally well be interpreted as the synthesis filter polyphase matrix. Therefore, the synthesis filters 


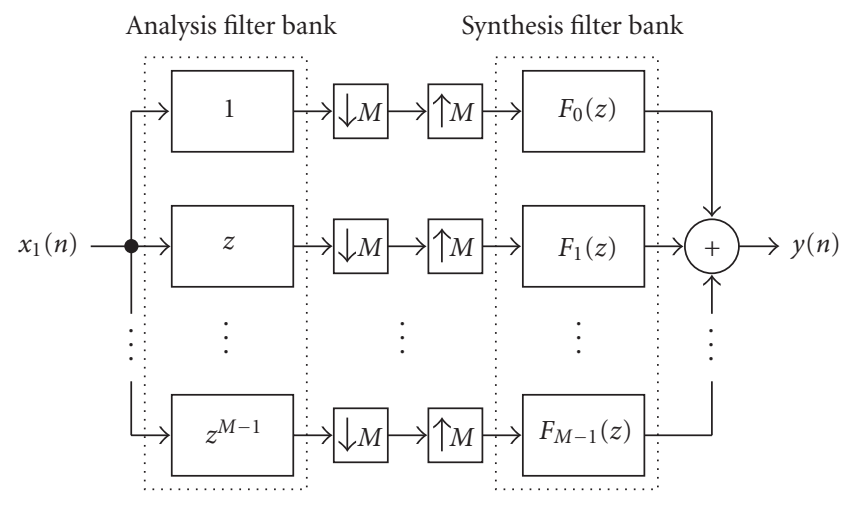

FIGURE 11: Equivalent filter bank structure to that in Figure 9 when $F_{n}(z), n=0,1, \ldots, M-1$, satisfy (53).

are obtained as

$$
\left[\begin{array}{c}
F_{0}(z) \\
F_{1}(z) \\
\vdots \\
F_{M-1}(z)
\end{array}\right]=\mathbf{R}^{T}\left(z^{M}\right)\left[\begin{array}{c}
1 \\
z^{-1} \\
\vdots \\
z^{-(M-1)}
\end{array}\right] .
$$

\subsection{Bunched nonuniform sampling}

A special case of periodic nonuniform sampling is what is referred to as bunched nonuniform sampling (exemplified in Figure 12), where the samples can be grouped into "uniform bunches" of $M$ samples taken with the higher sampling rate $1 / T_{1}$ within each bunch of duration $(M-1) T_{1}$ [12]. If the time duration between each pair of bunches of samples is chosen appropriately, the nonuniform-sampling sequence $x_{1}(n)$ can alternatively be obtained (or viewed as if obtained) through unconventional decimation of the oversampled uniform-sampling sequence $x_{2}(n)=x_{a}\left(n T_{1}\right)$, where $T_{1}=T /(1+P / M)$ and $P$ denotes the number of "missing samples" in $x_{2}(n)$ between each pair of adjacent bunches of samples contained in $x_{1}(n)$. Provided that $x_{2}(n)$ is bandlimited according to $\omega_{0} T_{1}<[1 /(1+P / M)] \pi$, which corresponds to $\omega_{0} T<\pi, x_{2}(n)$ can be recovered from $x_{1}(n)$ through the use of multirate $M$-channel FBs with a downsampling factor of $P+M$, trivial analysis filters (pure delays), and appropriately chosen synthesis filters $[22,23]$. However, in cases where it is desired to recover $x(n), x_{2}(n)$ must then be decimated by the rational factor $M / P$ which introduces the need for an additional filter. Using instead the time-varying filter approach in this paper, this problem is circumvented as $x(n)$ is obtained directly from $x_{1}(n)$. Furthermore, the timevarying filter approach has the additional advantage that it can handle the more general case, where the time duration between the bunches of samples is arbitrary, that is, when $x_{1}(n)$ no longer can be obtained from $x_{2}(n)$ through simple downsampling. This case cannot be handled by the FB approaches in $[22,23]$. The methods in $[22,23]$ also have some drawbacks that are overcome by using the time-varying filter approach. The technique in [22] cannot completely control
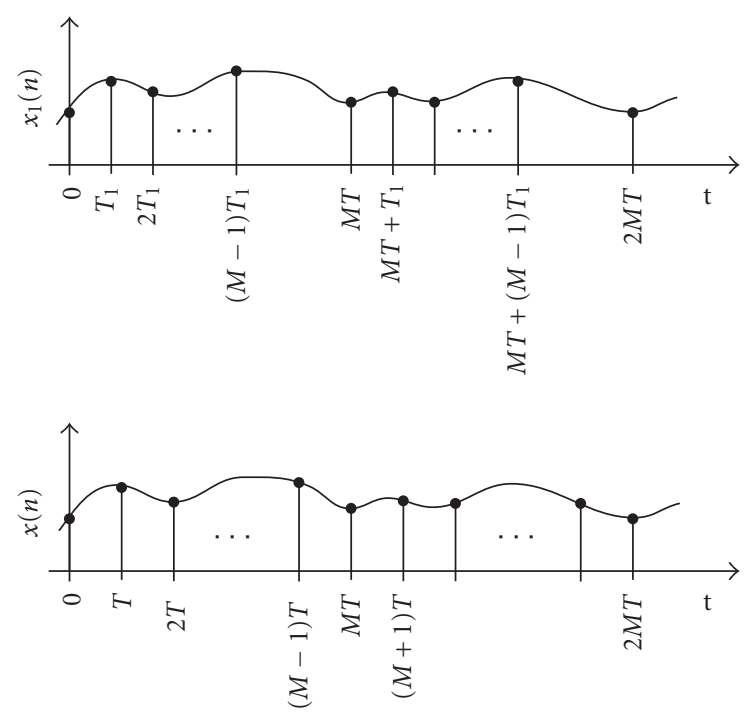

FIGURE 12: Illustration of bunched nonuniform sampling and reconstruction.

the aliasing components, whereas the approach in [23] requires a certain amount of additional oversampling.

Using the reconstruction technique considered in this paper, bunched nonuniform sampling is handled by selecting $\varepsilon_{n} T=\varepsilon_{n+M} T$ appropriately. An example will be provided later in Section 5.4.

\subsection{Filter design}

In the general case of nonuniform sampling, it is required to redesign $A_{n}(j \omega T)$ for each value of $n$, as discussed in Section 2.4. In a real-time application, there is thus a need to incorporate a processor that performs online design. In that case, it is crucial to make use of a closed form design technique, as other online optimization techniques are costly and time-consuming, albeit possible in principle. For this purpose, the least-square design technique introduced earlier in Section 4 is appropriate. This technique may be suitable also for the special case of periodically nonuniformly sampled signals even though it here suffices to design only $M$ ( $M$ being the periodicity) different filters as $A_{n}(j \omega T)=$ $A_{n+M}(j \omega T)$. One reason is that the application at hand may be appropriately designed by minimizing the error power (like in Section 3). Another reason is that it may be the case that $\varepsilon_{n} T$ may vary slightly with time even if they within different segments of samples may be considered fixed. In this case, it is again necessary to perform online design, although not for every output sample. One may then conveniently make use of least-square techniques to obtain slightly overdesigned filters, as discussed earlier in Section 2.4.

On the other hand, in cases where one knows that $\varepsilon_{n} T$ is periodic and fixed, and will not change, there is more freedom in choosing design methods and objective functions, as it then suffices to design $M$ filters beforehand. One such case occurs in reconstruction from bunched samples 
(see Example 5 in the next subsection). If the application at hand "requires" minimax design rather than least-square design, offline minimax design can be used to obtain the minimum-order filters for a given tolerable approximation error. For example, in communication applications, it is often important to suppress spurious components in which case the maximum of undesired frequency components must be kept below an acceptable level. In this case, the goal is to determine the $M$ impulse responses $h_{n}(k), n=0,1, \ldots, M-$ 1 , so that the distortion function $V_{0}\left(e^{j \omega T}\right)$ and aliasing functions $V_{m}\left(e^{j \omega T}\right), m=1,2, \ldots, M-1$, approximate one and zero, respectively, as close as desired in the minimax (Chebyshev) sense. From previous discussions in this section, and well-known input-output relations in maximally decimated FBs [21], it follows that the distortion and aliasing functions are given here by

$$
\begin{aligned}
V_{0}\left(e^{j \omega T}\right) & =\frac{1}{M} \sum_{n=0}^{M-1} G_{n}\left(e^{j \omega T}\right), \\
V_{m}\left(e^{j \omega T}\right) & =\frac{1}{M} \sum_{n=0}^{M-1} e^{-j 2 \pi m n / M} G_{n}\left(e^{j(\omega T-2 \pi m / M)}\right),
\end{aligned}
$$

where $G_{n}\left(e^{j \omega T}\right)$ are the periodic extensions of $A_{n}(j \omega T)$ in (10). The output Fourier transform can now be written as

$$
\begin{aligned}
Y\left(e^{j \omega T}\right)= & V_{0}\left(e^{j \omega T}\right) X\left(e^{j \omega T}\right) \\
& +\sum_{m=1}^{M-1} V_{m}\left(e^{j \omega T}\right) X\left(e^{j(\omega T-2 \pi m / M)}\right) .
\end{aligned}
$$

Perfect reconstruction $(\mathrm{PR})$ is obtained when $V_{0}\left(e^{j \omega T}\right)=$ 1 and $V_{m}\left(e^{j \omega T}\right)=0, m=1,2, \ldots, M-1$, in which case $Y\left(e^{j \omega T}\right)=X\left(e^{j \omega T}\right)$ and thus $y(n)=x(n)$. Whereas PR can be obtained in general practical FBs containing both free analysis and synthesis filters [21], only near-PR FBs can be achieved in our case. The reason is that the synthesis filters are fixed and trivial. Below, it is shown how to design the FB in the minimax sense. Minimax design: in ordinary near-PR FBs, it is customary to minimize the stopband energy of the channel filters subject to the constraints that the distortion and aliasing functions approximate one and zero, respectively, with the tolerances $\delta_{0}$ and $\delta_{1}$ according to

$$
\begin{aligned}
& \left|V_{0}\left(e^{j \omega T}\right)-1\right| \leq \delta_{0}, \quad \omega T \in \Omega_{0}, \\
& \left|V_{m}\left(e^{j \omega T}\right)-1\right| \leq \delta_{1}, \quad \omega T \in \Omega_{m},
\end{aligned}
$$

for $m=1,2, \ldots, M-1$ [21] and the frequency bands of interest, $\Omega_{0}$ and $\Omega_{m}$ (see below). Here, there are no channel filter requirements as the $\mathrm{FB}$ formulation is merely a convenient way of describing the reconstruction. This means that the design problem reduces to that of satisfying (57) which are minimax constraints. Furthermore, since $x_{a}(t)$ is assumed to be slightly oversampled, the region $\omega T \in[-\pi, \pi]$ incorporates don't-care bands where there are no requirements on $V_{0}\left(e^{j \omega T}\right)$ and $V_{m}\left(e^{j \omega T}\right)$. Consequently, the reconstruction filters can be obtained by solving the following minimax optimization problem.
Given the period $M$ and the subfilter's order $2 N$, find the unknown coefficients $h_{n}(k), k=-N,-N+1, \ldots, N, n=$ $0,1, \ldots, M-1$, and $\delta$, to minimize $\delta$ subject to

$$
\begin{aligned}
& \left|V_{0}\left(e^{j \omega T}\right)-1\right| \leq \delta, \quad \omega T \in \Omega_{0}, \\
& \left|V_{m}\left(e^{j \omega T}\right)-1\right| \leq \delta\left(\delta_{1} / \delta_{0}\right), \quad \omega T \in \Omega_{m}
\end{aligned}
$$

for $m=1,2, \ldots, M-1$, where

$$
\begin{aligned}
& \Omega_{0}=\left[-\omega_{0} T, \omega_{0} T\right], \\
& \Omega_{m}=\left[-\omega_{0} T+\frac{2 \pi m}{M}, \omega_{0} T+\frac{2 \pi m}{M}\right] .
\end{aligned}
$$

Clearly, $V_{0}\left(e^{j \omega T}\right)$ and $V_{m}\left(e^{j \omega T}\right)$ satisfy $(57)$ and (58), respectively, if $\delta$ after the optimization satisfies $\delta \leq \delta_{0}$.

In regular FBs, (60) would comprise nonlinear constraints because $V_{0}(z)$ and $V_{m}(z)$ then contain terms like $G_{n}(z) F_{n}(z)$, with $G_{n}(z)$ and $F_{n}(z)$ containing free parameters. This is not the case here as $V_{0}(z)$ and $V_{m}(z)$ in (54) and (55) only contain simple terms $G_{n}(z)$ and shifted versions thereof. This is due to the fact that $F_{n}(z)=z^{-n}$ are fixed to pure delays, and it implies that the above stated optimization problem is a convex problem which has a unique optimum solution. This solution can be obtained using standard techniques to this end. For example, by discretizing the problem, and making use of the real rotation theorem [24], the optimum solution can be found by solving a finite-dimensional linear programming problem [19].

Although the problem is convex, which guarantees that the obtained solution is the global optimum provided the optimization routine converges, it is often advantageous to start with a good initial solution. In this way, the speed as well as convergence may be improved. The solution provided by the least-square design technique in Section 4 is a good starting point for this purpose as least-square designs are rather close to minimax designs. This will be demonstrated in the example section below.

Finally, it is also mentioned that one naturally may consider constrained least-square optimization as an alternative to least-square and minimax optimization. This is a mixture of least-square and minimax optimization which may be more appropriate in some applications. By formulating the problem as a quadratic programming problem, a unique optimum solution can again be guaranteed.

\subsection{Design examples}

This subsection illustrates through design examples some features of the least-square and minimax design methods for the periodic nonuniform sampling case.

Example 5. A bunched-sample example taken from [12] is considered. In this example, the samples are taken at twice the average sampling rate $1 / T$, with bunches of five samples. 

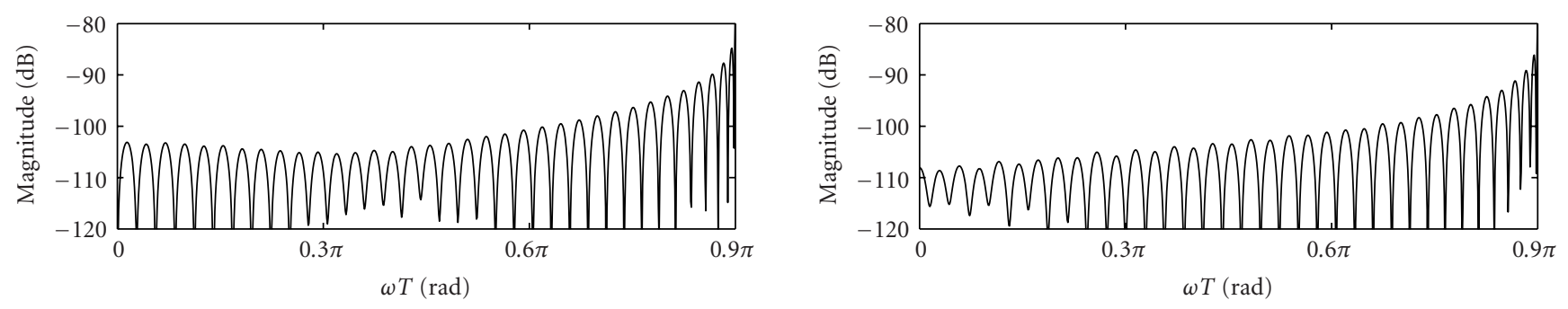

FIgURE 13: Magnitude of $A_{3}(j \omega T)-1$ and $A_{4}(j \omega T)-1$ in Example 5.

With the notation used above in Section 5.2, this means that $M=5, P=5$, and $T_{1}=T / 2$. In other words, the samples can be (viewed as if they were) obtained from a twotime oversampled signal through unconventional decimation. Five consecutive samples are extracted, the following five samples are discarded, and so on. With the notation in (2), this sampling pattern corresponds to the following values of $\varepsilon_{n} T=\varepsilon_{n+5} T: \varepsilon_{0} T=0, \varepsilon_{1} T=-0.5 T, \varepsilon_{2} T=-T$, $\varepsilon_{3} T=-1.5 T, \varepsilon_{4} T=-2 T$.

It is noted here that the first, third, and fifth samples in each bunch of samples are actually the first, second, and third sample in the desired sequence. This means that $h_{n}(k)$ for $n=0,1,2$, correspond to "negative delays" of $-n$; that is, $h_{n}(k)=\delta(k+n)$, where $\delta(n)$ denotes the unit impulse sequence. Designing for $\omega_{0} T=0.9 \pi$, the remaining two filters, $h_{n}(k), n=3,4$, using the least-square approach in Section 4, and a filter order of 70, an SNR (see Footnote 5) of about $104 \mathrm{~dB}$ is reached. ${ }^{9}$ In [12], filter orders of 80 are employed to reach an SNR of about $100 \mathrm{~dB}$. Thus, using the proposed design technique, the filter order can be reduced.

Figure 13 plots the magnitudes of $A_{3}(j \omega T)-1$ and $A_{4}(j \omega T)-1$. Figure 14 plots the magnitudes of $V_{0}\left(e^{j \omega T}\right)-1$ and $V_{m}\left(e^{j \omega T}\right), m=1,2,3,4$. Figure 15 plots the magnitude responses of the corresponding synthesis filters $F_{n}\left(e^{j \omega T}\right), n=$ $0,1,2,3,4$, that are related to $H_{n}\left(e^{j \omega T}\right)$ through $(51)-(53)$. It is interesting to note that $F_{n}(z)$ are multilevel filters as concluded in [7]. However, in this paper, the multilevel filters are not designed explicitly as $H_{n}(z)$ are obtained in the design process. The multilevel filters are then obtained from $H_{n}(z)$ through (51)-(53).

Example 6. In Figure 14, it is noted that $V_{0}\left(e^{j \omega T}\right)$ and $V_{m}\left(e^{j \omega T}\right)$ exhibit "least-square behavior," which is due to the fact that $G_{n}(z)$ in Example 5 above were obtained using the least-square approach in Section 4 . The largest errors are found at the signal band (passband) edges which are typical for least-square design techniques [8]. If it is desired to suppress the largest errors and instead obtain a minimax solution, one can use the least-square solution as a starting point for optimization in the minimax sense, as discussed above in

\footnotetext{
${ }^{9}$ This has been verified through simulation with colored noise as input signal (constant power spectrum in the frequency region $\left[-\omega_{0} T, \omega_{0} T\right]$ ).
}

Section 5.3. With $\delta_{0}=\delta_{1}$ in (57) and (58), the resulting magnitude responses are as shown in Figure 16. It is seen that the errors now exhibit "minimax behavior," and it turns out that the maximum of the errors has been decreased by some $6 \mathrm{~dB}$ in this example.

Example 7. The example in [13] is considered which deals with time-skew errors in time-interleaved ADCs. In this example, the CT input signal is a sum of four sinusoidals with angular frequencies $0.125 \pi / T, 0.25 \pi / T, 0.375 \pi / T$, and $0.5 \pi / T$, respectively. Furthermore, the number of channels is $M=5$, and the sampling instance errors $\varepsilon_{n} T, n=0,1,2,3,4$, in (2), are assumed to be $0,-0.04 T, 0.02 T,-0.01 T$, and $0.03 T$, respectively. The obtained nonuniform-sampling sequence $x_{1}(n)$ has a spectrum according to Figure 17. Apparently, several undesired frequency components with large amplitudes have been introduced due to the nonuniform sampling. In [13], the distortion was reduced by using a bank of $M$ fractional-delay FIR filters of order eight which suppress the undesired components to some $80 \mathrm{~dB}$. Using instead the proposed approach with eighth-order filters designed for the same bandwidth, namely, $\omega_{0} T=0.6 \pi$, about the same performance is obtained as seen in Figure 18, which plots the spectrum of the reconstructed sequence $y(n) .{ }^{10}$

Example 8. A drawback of the method in [13] is that an additional amount of oversampling must be used. In the example above, the upper limit is $\omega_{0} T=0.6 \pi$. Using instead the general time-varying filters considered here, this problem is overcome. As demonstrated earlier in Section 4, one can let $\omega_{0} T$ be rather close to $\pi$ and still handle this in practice. For example, with $\omega_{0} T=0.9 \pi$, and 44 th-order filters, an SNR of more than $100 \mathrm{~dB}$ is achieved. Applying these filters to a nonuniformly sampled multi-sine signal with angular frequencies $0.125 \pi / T, 0.25 \pi / T, 0.375 \pi / T$, $0.5 \pi / T, 0.625 \pi / T, 0.75 \pi / T$, and $0.875 \pi / T$, and with the

\footnotetext{
10 As discussed in Section 2.5, one can achieve perfect reconstruction when the input is a multi-sine signal, provided that the frequencies are known. However, the reconstruction filters are here designed to handle general bandlimited signals. This is what one usually has to do in practice anyway as it may be difficult to know exactly the frequencies. A typical example of this is found in communication systems where unknown frequency offsets are present [25].
} 

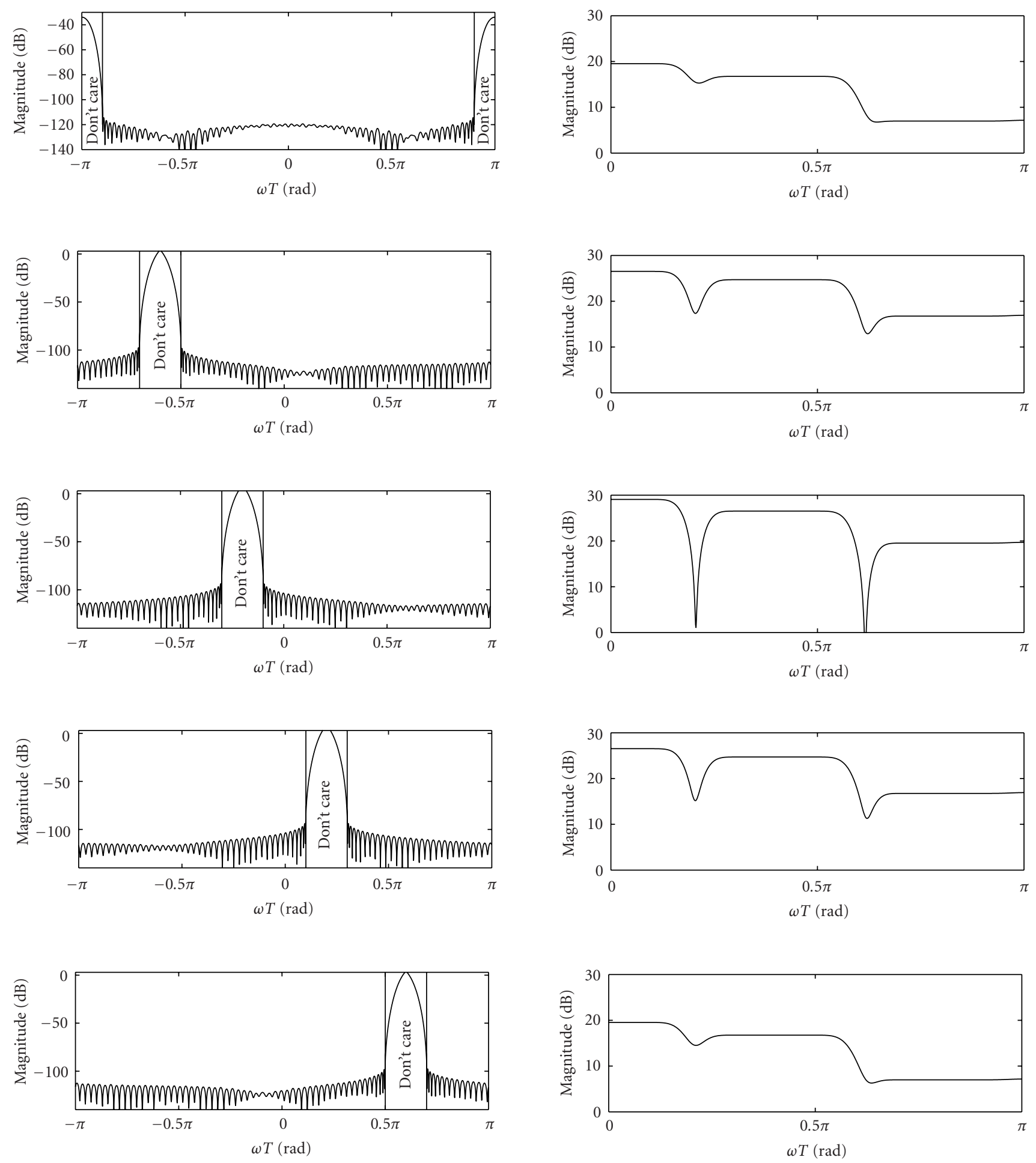

FIgURe 14: Magnitude of $V_{0}\left(e^{j \omega T}\right)-1$ (top) and $V_{m}\left(e^{j \omega T}\right), m=$ 1,2,3, 4, in Example 5.

same sampling pattern as in Example 7, the result of which is the sequence $x_{1}(n)$ with spectrum according to Figure 19, the spectrum of the reconstructed sequence $y(n)$ becomes as shown in Figure 20. It should be pointed out, however, that the approach in [13] has the advantage that the

fractional-delay filters can be implemented in such a way (using Farrow-based structures [26, 27]) that online design can be avoided when the sampling instance errors are changed. Using the general time-varying filters, online design is inevitable which increases the implementation cost. On the 

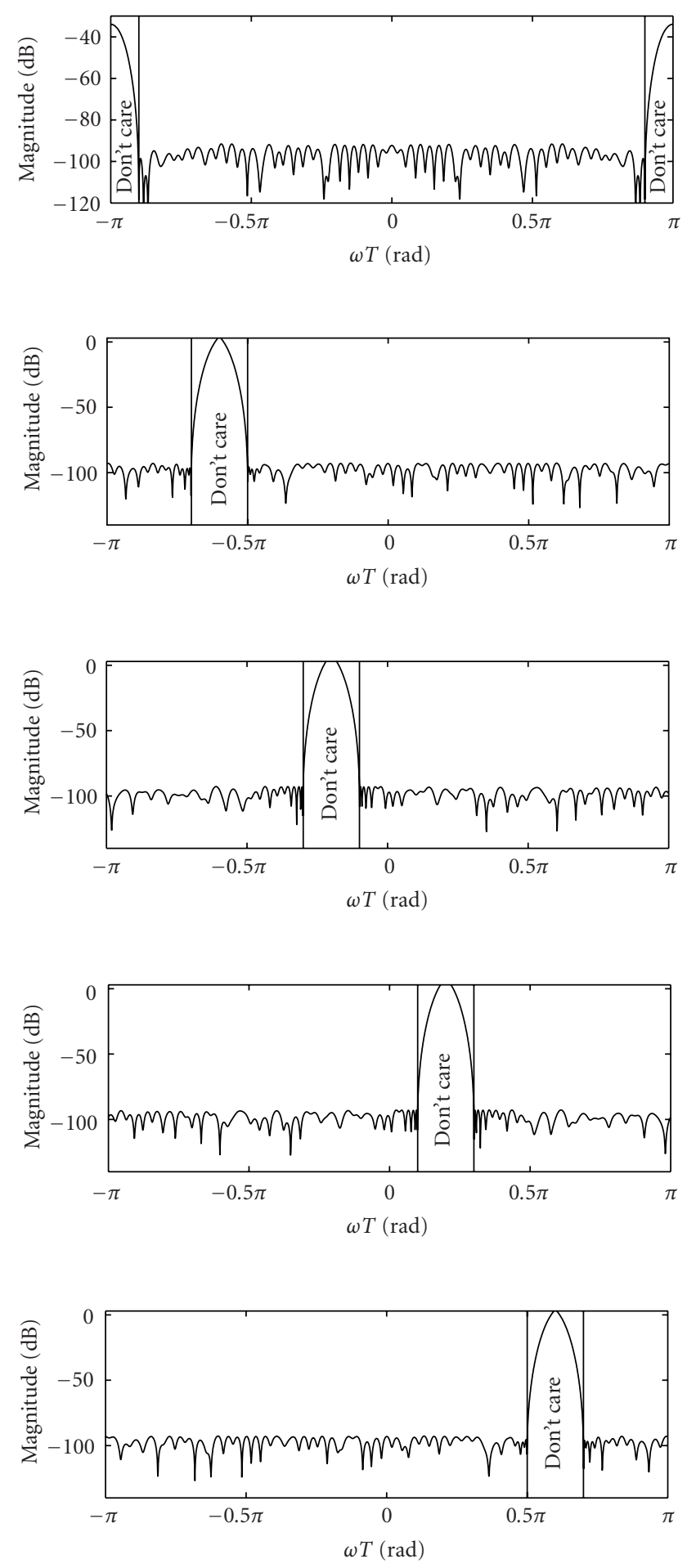

Figure 16: Magnitude of $V_{0}\left(e^{j \omega T}\right)-1$ (top) and $V_{m}\left(e^{j \omega T}\right), m=$ 1,2,3, 4, in Example 6.

other hand, the general filters can handle wider bandwidths so which one of the two methods to use for reconstructing periodically nonuniformly sampled signals largely depends on the application at hand.

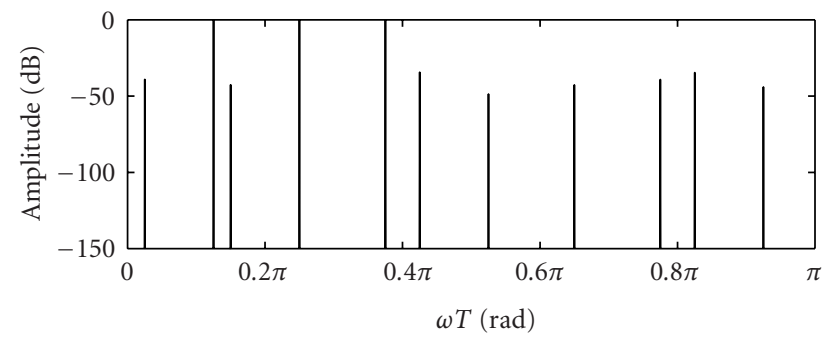

FIGURE 17: Spectrum of the sequence $x_{1}(n)$ in Example 7.

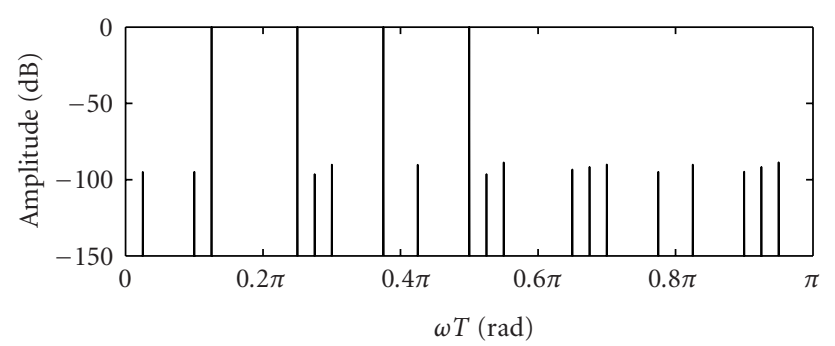

FIGURE 18: Spectrum of the reconstructed sequence $y(n)$ in Example 7.

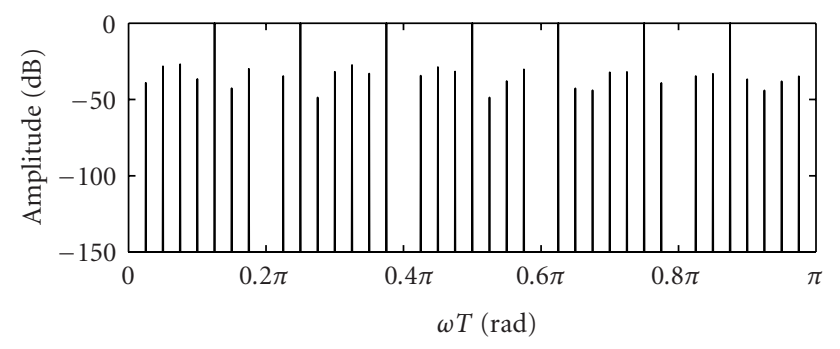

FIGURE 19: Spectrum of the sequence $x_{1}(n)$ in Example 8 .

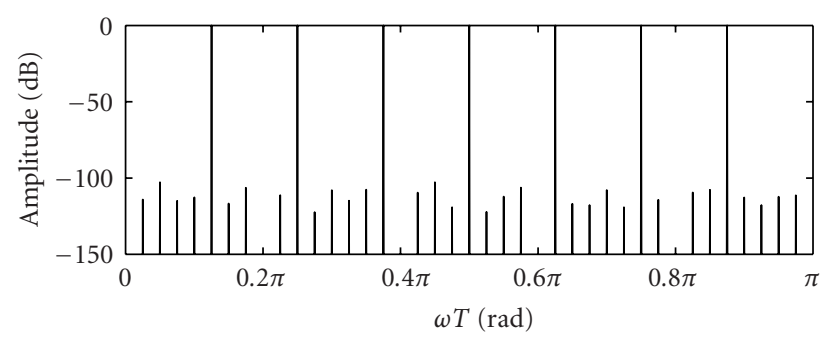

FIgURE 20: Spectrum of the reconstructed sequence $y(n)$ in Example 8.

Finally, it is noted that the same example is considered in [12], where the filters $F_{n}(z)$ are of order 149. Hence, using the proposed design approach, the complexity can be reduced. 


\section{EXTENSION TO BANDPASS SIGNALS}

This paper has so far been concerned with lowpass signals bandlimited according to (3) (see also Figure 2). It is, however, possible to extend the results to bandpass signals after appropriate modifications. The extensions are rather straightforward so this section will merely give the necessary modifications.

Consider the case where the signal $x_{a}(t)$ is a bandpass signal bandlimited to the frequency region $|\omega| \in\left[\omega_{0}, \omega_{1}\right]$, where $\omega_{0} \geq 0, \omega_{1}<\pi / T$, and $\omega_{0}<\omega_{1}$. In principle, the reconstruction is conducted in the same way as for the lowpass case. The only difference is that $A_{n}(j \omega T)$ in (10) now should approximate one in the frequency region $|\omega T| \in$ $\left[\omega_{0} T, \omega_{1} T\right]$. Using the least-square design technique introduced in Section 4, this amounts to replacing (44) and (45) with

$$
\begin{gathered}
c_{n, k}=\left\{\begin{array}{l}
-\frac{2\left(\omega_{1} T-\omega_{0} T\right)}{\pi}, \quad k-\varepsilon_{n-k}=0, \\
-\frac{2 \sin \left[\omega_{1} T\left(k-\varepsilon_{n-k}\right)\right]}{\pi\left(k-\varepsilon_{n-k}\right)} \\
\quad+\frac{2 \sin \left[\omega_{0} T\left(k-\varepsilon_{n-k}\right)\right]}{\pi\left(k-\varepsilon_{n-k}\right)}, \quad k-\varepsilon_{n-k} \neq 0
\end{array}\right. \\
s_{n, k p}=\left\{\begin{array}{l}
\frac{\omega_{1} T-\omega_{0} T}{\pi}, \quad k=p, \\
\frac{\sin \left[\omega_{1} T\left(k+\varepsilon_{n-p}-p-\varepsilon_{n-k}\right)\right]}{\pi\left(k+\varepsilon_{n-p}-p-\varepsilon_{n-k}\right)} \\
-\frac{\sin \left[\omega_{0} T\left(k+\varepsilon_{n-p}-p-\varepsilon_{n-k}\right)\right]}{\pi\left(k+\varepsilon_{n-p}-p-\varepsilon_{n-k}\right)}, \quad k \neq p
\end{array}\right.
\end{gathered}
$$

respectively. Likewise, when using minimax design as outlined in Section 5, it amounts to replacing (61) and (62) with the appropriate frequency regions.

More generally, $x_{a}(t)$ is a bandpass signal bandlimited to the frequency region $|\omega| \in\left[\omega_{0}, \omega_{1}\right]$, where $\omega_{0} \geq 2 \pi p / T$, $\omega_{1}<\pi / T+2 \pi p / T$, and $\omega_{0}<\omega_{1}$, with $p$ being a positive integer. This case is handled in the same way as above. Hence, as to the least-square design, (63) and (64) still apply. The only difference is that $\omega_{0}$ and $\omega_{1}$ take on different values.

\subsection{Design examples}

Although the reconstruction in principle is done in the same way as for the lowpass case, we have observed experimentally that the filter order required to achieve a certain SNR for a fixed bandwidth is generally higher in the bandpass case than in the lowpass case. This is exemplified in the examples below.

Example 9. Figure 21 plots the magnitudes of $A_{n}(j \omega T)-1$ for the same sampling pattern and filter orders as used earlier in Example 1. Comparing Figure 21 with Figure 3, it is seen that the reconstruction error is larger in the bandpass case. The band edges are here $\omega_{0} T=0.05 \pi$ and $\omega_{1} T=0.95 \pi$.

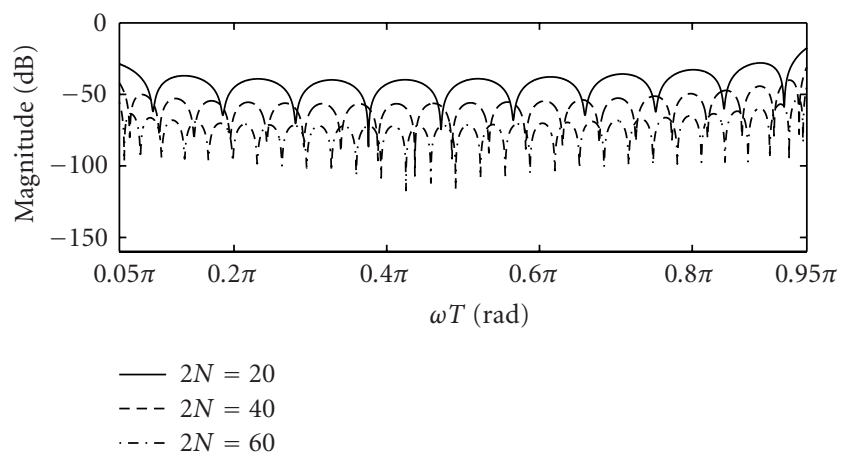

Figure 21: Magnitude $\left(20 \log _{10}\left|A_{n}-1\right|\right.$ for even $\left.n\right)$ for the proposed least-square filters of order $2 \mathrm{~N}$ for bandpass signals (Example 9).

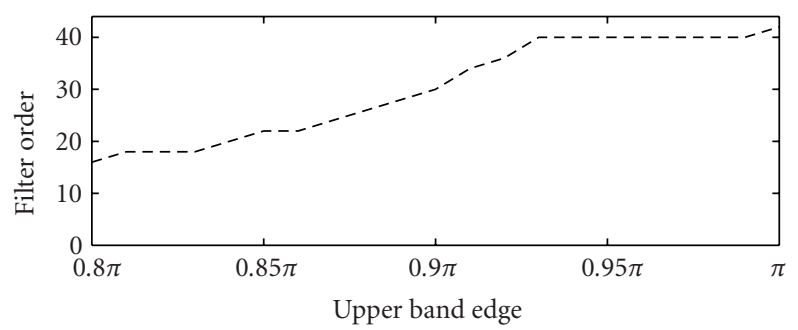

Figure 22: Filter order versus upper band edge for a bandwidth of $0.8 \pi$ and reconstruction error of $-60 \mathrm{~dB}$ (Example 10).

Example 10. Figure 22 plots the filter order as a function of the upper band edge $\omega_{1} T$ for a fixed bandwidth of $\omega_{1} T-$ $\omega_{0} T=0.8 \pi$. (The same sampling pattern and randomization as those employed in Examples 6 and 7 were used.) It is seen that the filter order increases with the upper band edge $\omega_{1} T$. Thus, from a complexity point of view, the lowpass case is advantageous.

\section{CONCLUDING REMARKS}

The main theme of this paper has been to show how a slight oversampling can be properly utilized in the design of timevarying discrete-time FIR filters for reconstructing nonuniformly sampled signals. As has been demonstrated through several design examples, one can in this way obtain as small as desired reconstruction error by simply increasing the filter order. This has been exemplified both for the general case and for periodically nonuniformly sampled signals. Furthermore, both least-square and minimax design have been discussed.

As the underlying assumption is bandlimitation, problems may arise though as signals are usually not strictly bandlimited in practice. In particular, when the sampling is "far from uniform", like bunched sampling, the magnitude of the filter coefficients $h_{n}(k)$ tends to be rather large. This means, in particular, that if noise is present in the frequency region $|\omega T| \in\left(\omega_{0} T, \pi\right]$ (in the lowpass case), due to inevitable data quantization and so forth, the noise contained in the reconstructed sequence $y(n)$ may become 
rather large. ${ }^{11}$ This is, however, due to the fact that "the more nonuniform" the sampling is, the more ill-conditioned the reconstruction problem is [1]. It is thus not a problem associated with the reconstruction method studied in this paper. Sampling patterns with small deviations from the uniform grid, like those occurring in time-interleaved ADCs, are not associated with such problems. In such cases, all filter coefficients $h_{n}(k)$ are small in magnitude, except for the middle tap that is close to one. ${ }^{12}$ The noise in the reconstructed sequence $y(n)$ will then be more or less the same as in the uniformsampling sequence $x(n)$.

Another practical problem is the quantization of the filter coefficients $h_{n}(k)$. This problem can, however, always be solved by allocating more bits to $h_{n}(k)$. Coefficient quantization is thus not a limiting factor as to the quality of the reconstruction. Furthermore, as FIR filters are employed, the coefficient sensitivity of the reconstruction filters is similar to that of regular FIR filters which are known to have a relatively low sensitivity.

\section{REFERENCES}

[1] F. Marvasti, Ed., Nonuniform Sampling: Theory and Practice, Kluwer Academic, New York, NY, USA, 2001.

[2] W. C. Black and D. A. Hodges, "Time interleaved converter arrays," IEEE Journal of Solid-State Circuits, vol. 15, no. 6, pp. 1022-1029, 1980.

[3] J. L. Yen, "On nonuniform sampling of bandwidth-limited signals," IRE Transactions on Circuit Theory, vol. 3, no. 4, pp. 251257, 1956.

[4] A. J. Jerri, "The Shannon sampling theorem-its various extensions and applications: a tutorial review," Proceedings of IEEE, vol. 65, no. 11, pp. 1565-1596, 1977.

[5] A. Papoulis, "Generalized sampling expansion," IEEE Transactions on Circuits and Systems, vol. 24, no. 11, pp. 652-654, 1977.

[6] H. Choi and D. C. Munson, "Analysis and design of minimax optimal interpolators," IEEE Transactions on Signal Processing, vol. 46, no. 6, pp. 1571-1579, 1998.

[7] Y. C. Eldar and A. V. Oppenheim, "Filterbank reconstruction of bandlimited signals from nonuniform and generalized samples," IEEE Transactions on Signal Processing, vol. 48, no. 10, pp. 2864-2875, 2000.

[8] T. Saramäki, "Finite impulse response filter design," in Handbook for Digital Signal Processing, S. K. Mitra and J. F. Kaiser, Eds., chapter 4, pp. 155-277, John Wiley \& Sons, New York, NY, USA, 1993.

[9] H. Jin and E. K. F. Lee, "A digital-background calibration technique for minimizing timing-error effects in time-interleaved ADCs," IEEE Transactions on Circuits and Systems II: Analog and Digital Signal Processing, vol. 47, no. 7, pp. 603-613, 2000.

${ }^{11}$ If the noise is colored and bandlimited to $\omega_{0} T$, the noise in $y(n)$ will not blow up in the reconstruction process. This is explained by noting the fact that the noise in that case can be viewed as "correctly" reconstructed CT colored noise bandlimited to $\omega_{0}$.

12 When $t_{n}=n T, g_{n}(0)=1$, whereas the remaining $g_{n}(k)$ are zero, as seen in (10).
[10] J. Higgins, "A sampling theorem for irregularly spaced sample points (Corresp.)," IEEE Transactions on Information Theory, vol. 22, no. 5, pp. 621-622, 1976.

[11] W. Namgoong, "Finite-length synthesis filters for nonuniformly time-interleaved analog-to-digital converter," in Proceedings of IEEE International Symposium on Circuits and Systems (ISCAS '02), vol. 4, pp. 815-818, Phoenix-Scottsdale, Ariz, USA, May 2002.

[12] R. S. Prendergast, B. C. Levy, and P. J. Hurst, "Reconstruction of bandlimited periodic nonuniformly sampled signals through multirate filter banks," IEEE Transactions on Circuits and Systems I: Fundamental Theory and Applications, vol. 51, no. 12, pp. 1612-1622, 2004.

[13] H. Johansson and P. Löwenborg, "Reconstruction of nonuniformly sampled bandlimited signals by means of digital fractional delay filters," IEEE Transactions on Signal Processing, vol. 50, no. 11, pp. 2757-2767, 2002.

[14] H. Johansson and P. Löwenborg, "Reconstruction of nonuniformly sampled bandlimited signals using time-varying discrete-time FIR filters," in Proceedings of 12th European Signal Processing Conference (EUSIPCO '04), Vienna, Austria, September 2004.

[15] H. Johansson and P. Löwenborg, "Reconstruction of periodically nonuniformly sampled bandlimited signals using timevarying FIR filters," in Proceedings of 4th International Workshop Spectral Methods Multirate Signal Processing (SMMSP '04), Vienna, Austria, September 2004.

[16] N. Maeda, "Transversal filters with nonuniform tap spacings," IEEE Transactions on Circuits and Systems, vol. 27, no. 1, pp. $1-11,1980$.

[17] P. Jarske, T. Saramäki, S. K. Mitra, and Y. Neuvo, “On properties and design of nonuniformly spaced linear arrays [antennas]," IEEE Transactions on Acoustics, Speech, Signal Processing, vol. 36, no. 3, pp. 372-380, 1988.

[18] J. O. Coleman, "Choosing nonuniform tap spacings for a tapped-delay-line filter," IEEE Transactions on Circuits and Systems II: Analog and Digital Signal Processing, vol. 43, no. 4, pp. 298-303, 1996.

[19] S. G. Nash and A. Sofer, Linear and Nonlinear Programming, McGraw-Hill, New York, NY, USA, 1996.

[20] T. I. Laakso, V. Valimäki, M. Karjalainen, and U. K. Laine, "Splitting the unit delay [FIR/all pass filters design]," Signal Processing Magazine, vol. 13, no. 1, pp. 30-60, 1996.

[21] P. P. Vaidyanathan, Multirate Systems and Filter Banks, Prentice-Hall, Englewood Cliffs, NJ, USA, 1993.

[22] P. P. Vaidyanathan and V. C. Liu, "Efficient reconstruction of band-limited sequences from nonuniformly decimated versions by use of polyphase filter banks," IEEE Transactios on Acoustics, Speech, Signal Processing, vol. 38, no. 11, pp. 19271936, 1990.

[23] H. Johansson and P. Löwenborg, "Reconstruction of a class of nonuniformly sampled and decimated bandlimited signals," in Proceedings of IEEE International Symposium on Circuits and Systems (ISCAS '02), vol. 2, pp. 604-607, Phoenix-Scottsdale, Ariz, USA, May 2002.

[24] T. W. Parks and C. S. Burrus, Digital Filter Design, John Wiley \& Sons, New York, NY, USA, 1987.

[25] L. Hanzo, M. Münster, B. J. Choi, and T. Keller, OFDM and MC-CDMA for Broadband Multi-user Communications, WLANs and Broadcasting, John Wiley \& Sons, West Sussex, UK, 2003. 
[26] J. Vesma and T. Saramäki, "Optimization and efficient implementation of FIR filters with adjustable fractional delay," in Proceedings of IEEE International Symposium on Circuits and Systems (ISCAS '97), vol. 4, pp. 2256-2259, Hong Kong, June 1997.

[27] H. Johansson and P. Löwenborg, "On the design of adjustable fractional delay FIR filters," IEEE Transactions on Circuits and Systems II: Analog and Digital Signal Processing, vol. 50, no. 4, pp. 164-169, 2003.

Håkan Johansson was born in Kumla, Sweden, in 1969. He received the M.S. degree in computer science and the Licentiate, Doctoral, and Docent degrees in electronics systems from Linkoping University, Sweden, in 1995, 1997, 1998, and 2001, respectively. During 1998 and 1999, he held a post-doctoral position at Signal Processing Laboratory, Tampere University of Technology, Finland. He is currently Professor in

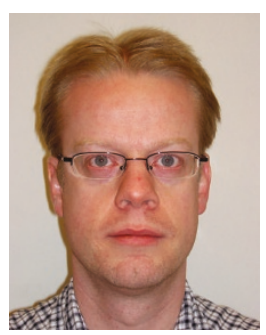
electronics systems at the Department of Electrical Engineering of Linkoping University. Professor Johansson's research interests include theory, design, and implementation of signal processing systems. He is the author or coauthor of four textbooks and about 100 international journal and conference papers. Professor Johansson has served as Associate Editor for IEEE Transactions on Circuits and Systems-II (2000-2001), and is currently Associate Editor for IEEE Signal Processing Letters, and a Member of the IEEE International Symposium on Circuits and Systems DSP Track Committee.

Per Löwenborg was born in Oskarshamn, Sweden, in 1974. He received the M.S. degree in applied physics and electrical engineering, and the Licentiate, and Doctoral degrees in electronics systems from Linkoping University, Sweden, in 1998, 2001, and 2002, respectively. Dr. Lowenborg's research interests are within the field of theory, de-

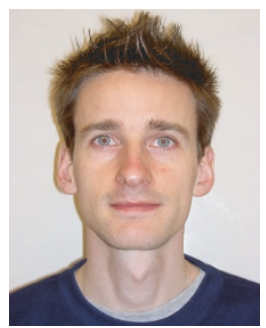
sign, and implementation of analog and digital signal processing electronics. He is the author or coauthor of one book and about 40 international journal and conference papers. He was awarded the 1999 IEEE Midwest Symposium on Circuits and Systems Best Student Paper Award and the 2002 IEEE Nordic Signal Processing Symposium Best Paper Award. Dr. Lowenborg is a Member of the IEEE. 